\title{
SUPPLEMENTARY MATERIAL FOR A LOSS FUNCTION APPROACH TO MODEL SPECIFICATION TESTING AND ITS RELATIVE EFFICIENCY
}

\author{
Yongmiao Hong \\ Yoon-Jin Lee \\ Cornell University 83 Xiamen University \\ Indiana University
}

This Supplementary Material contains two parts. Part A is the detailed proof of Theorems 1-4, and Part B reports the simulation results for the tests with the bandwidth $h=S_{X} n^{-1 / 5}$, where $S_{X}$ is the sample standard deviation of $\left\{X_{t}\right\}_{t=1}^{n}$.

A. Detailed Proof of Theorems 1-4. Throughout, we let $\tilde{m}_{h}(x)$ be defined in the same way as $\hat{m}_{h}(x)$ with $\left\{\varepsilon_{t}\right\}_{t=1}^{n}$ replacing $\left\{\hat{\varepsilon}_{t}\right\}_{t=1}^{n}$. Also, let $C \in(1, \infty)$ denote a generic bounded constant.

Proof of Theorem 1: Theorem 1 follows as a special case of Theorem $3(\mathrm{i})$ with $\delta\left(X_{t}\right)=0$.

Proof of Theorem 2: Theorem 2 follows as a special case of Theorem 3(ii) with $\delta\left(X_{t}\right)=0$.

Proof of Theorem 3(i): We shall first derive the asymptotic distribution of $q_{n}$ under $\mathbb{H}_{n}\left(a_{n}\right)$. From Lemmas 3.1 and 3.2 and Propositions 3.1 and 3.2 below, we can obtain

$$
\frac{h^{\frac{p}{2}} D^{-1} q_{n}-h^{-\frac{p}{2}} \sigma^{-2} \int K^{2}(u) d u \int \sigma^{2}(x) d x}{\sqrt{2 \sigma^{-4} \int\left[\int K(u) K(u+v) d u\right]^{2} d v \int \sigma^{4}(x) d x}} \stackrel{d}{\longrightarrow} N(\psi, 1) .
$$

The desired result of Theorem 3(i) then follows immediately.

Lemma 3.1: Under the conditions of Theorem 3, $\hat{Q}_{n}=D \sum_{t=1}^{n} \hat{m}_{h}^{2}\left(X_{t}\right)+o_{p}\left(h^{-p / 2}\right)$.

Lemma 3.2: Under the conditions of Theorem 3, $\sum_{t=1}^{n} \hat{m}_{h}^{2}\left(X_{t}\right)=n \int \hat{m}_{h}^{2}(x) f(x) d x+o_{p}\left(h^{-p / 2}\right)$.

Proposition 3.1: Under the conditions of Theorem 3, $n \int \hat{m}_{h}^{2}(x) f(x) d x=n \int \tilde{m}_{h}^{2}(x) f(x) d x+h^{-p / 2} E\left[\delta^{2}\left(X_{t}\right)\right]+$ $o_{p}\left(h^{-p / 2}\right)$.

Proposition 3.2: Under the conditions of Theorem 3 and $\mathbb{H}_{n}\left(a_{n}\right)$ with $a_{n}=n^{-1 / 2} h^{-p / 4}$,

$$
\left[n h^{p / 2} \int \tilde{m}_{h}^{2}(x) f(x) d x-h^{-\frac{p}{2}} a(K) \int \sigma^{2}(x) d x\right] / \sqrt{2 b(K) \int \sigma^{4}(x) d x} \stackrel{d}{\longrightarrow} N(\psi, 1) .
$$

Proof of Lemma 3.1: We put

$$
w_{s t}=\frac{\mathbf{K}_{h}\left(X_{t}-X_{s}\right)}{\sum_{t=1}^{n} \mathbf{K}_{h}\left(X_{t}-X_{s}\right)},
$$

and $\tilde{w}_{t s}=\frac{\mathbf{K}_{h}\left(X_{t}-X_{s}\right)}{n f\left(X_{t}\right)}$, where $f(\cdot)$ is the probability density function of $X_{t}$. Then we have $\sum_{t=1}^{n} w_{s t}=1$ for all $s$ and $\hat{m}_{h}\left(X_{t}\right)=\sum_{s=1}^{n} w_{s t} \hat{\varepsilon}_{s}$. By a second order Taylor series expansion, we have

$$
\begin{aligned}
\hat{Q}_{n} & =\sum_{t=1}^{n} d(0)+\sum_{t=1}^{n} d^{\prime}(0) \hat{m}_{h}\left(X_{t}\right)+\frac{1}{2} \sum_{t=1}^{n} d^{\prime \prime}(0) \hat{m}_{h}^{2}\left(X_{t}\right)+\frac{1}{2} \sum_{t=1}^{n}\left[d^{\prime \prime}\left(\bar{m}_{h}\left(X_{t}\right)\right)-d^{\prime \prime}(0)\right] \hat{m}_{h}^{2}\left(X_{t}\right) \\
& =\frac{d^{\prime \prime}(0)}{2} \sum_{t=1}^{n} \hat{m}_{h}^{2}\left(X_{t}\right)+\hat{R},
\end{aligned}
$$

given Assumption A.5, where $\bar{m}\left(X_{t}\right)$ lies between $\hat{m}_{h}\left(X_{t}\right)$ and 0 , and

$$
|\hat{R}|=\left|\frac{1}{2} \sum_{t=1}^{n}\left[d^{\prime \prime}\left(\bar{m}_{h}\left(X_{t}\right)\right)-d^{\prime \prime}(0)\right] \hat{m}_{h}^{2}\left(X_{t}\right)\right| \leq C \sum_{t=1}^{n}\left|\hat{m}_{h}^{3}\left(X_{t}\right)\right|
$$


given $\left|d^{\prime \prime}(z)-d^{\prime \prime}(0)\right| \leq C|z|$ in Assumption A.5. Furthermore, by the triangle inequality, we have

$$
\begin{gathered}
\sum_{t=1}^{n}\left|\hat{m}_{h}^{3}\left(X_{t}\right)\right| \leq 8 \sum_{t=1}^{n}\left|n^{-1} \sum_{s=1}^{n} \tilde{w}_{t s} \hat{\varepsilon}_{s}\right|^{3}+8 \sum_{t=1}^{n}\left|n^{-1} \sum_{s=1}^{n}\left(w_{t s}-\tilde{w}_{t s}\right) \hat{\varepsilon}_{s}\right|^{3} \\
=8 \sum_{t=1}^{n}\left|\sum_{s=1}^{n} \tilde{w}_{t s} \hat{\varepsilon}_{s}\right|^{3}+\sum_{t=1}^{n}\left|n^{-1} \sum_{s=1}^{n} \hat{\varepsilon}_{s}\right|^{3} O_{p}\left(\sup _{x \in \mathbb{G}}|\hat{f}(x)-f(x)|\right) .
\end{gathered}
$$

We only need to consider the order of magnitude for the first term in (A.3), since the second term will be a small order term given $\sup _{x \in \mathbb{G}}|\hat{f}(x)-f(x)| \stackrel{p}{\rightarrow} 0$. Define $\varepsilon_{n t}=Y_{t}-g\left(X_{t}, \theta_{0}\right)$. Then $\varepsilon_{n t}=a_{n} \delta\left(X_{t}\right)+\varepsilon_{t}$ under $\mathbb{H}_{n}\left(a_{n}\right)$. Hence, from $\hat{\varepsilon}_{t}=\left(\hat{\varepsilon}_{t}-\varepsilon_{n t}\right)+\left(\varepsilon_{n t}-\varepsilon_{t}\right)+\varepsilon_{t}$, we can decompose

$$
\begin{aligned}
\sum_{t=1}^{n}\left|n^{-1} \sum_{s=1}^{n} \hat{\varepsilon}_{s} \mathbf{K}_{h}\left(X_{t}-X_{s}\right)\right|^{3} \leq & 8 \sum_{t=1}^{n}\left|n^{-1} \sum_{s=1}^{n}\left(\hat{\varepsilon}_{s}-\varepsilon_{n s}\right) \mathbf{K}_{h}\left(X_{t}-X_{s}\right)\right|^{3} \\
& +8 \sum_{t=1}^{n}\left|n^{-1} \sum_{s=1}^{n}\left(\varepsilon_{n s}-\varepsilon_{s}\right) \mathbf{K}_{h}\left(X_{t}-X_{s}\right)\right|^{3}+8 \sum_{t=1}^{n}\left|n^{-1} \sum_{s=1}^{n} \varepsilon_{s} \mathbf{K}_{h}\left(X_{t}-X_{s}\right)\right|^{3} \\
= & 8\left(\hat{R}_{1}+\hat{R}_{2}+\hat{R}_{3}\right), \text { say. }
\end{aligned}
$$

First, we consider the $\hat{R}_{1}$ term in (A.4). Given $\hat{\varepsilon}_{s}-\varepsilon_{n s}=g\left(X_{t}, \theta_{0}\right)-g\left(X_{t}, \hat{\theta}_{0}\right)$, we have

$$
\begin{aligned}
\hat{R}_{1} & \leq\left\|\hat{\theta}-\theta_{0}\right\|^{3} \sum_{t=1}^{n}\left\|n^{-1} \sum_{s=1}^{n} \nabla_{\theta} g\left(X_{t}, \bar{\theta}\right) \mathbf{K}_{h}\left(X_{t}-X_{s}\right)\right\|^{3} \\
& =n O_{p}\left(n^{-3 / 2} h^{-2 p}\right)=o_{p}\left(h^{-p / 2}\right),
\end{aligned}
$$

by the mean value theorem and the condition that $h \propto n^{-\omega}$ for $\omega \in(0,1 / 2 p)$.

Next, for the $\hat{R}_{2}$ term in (A.4), we have

$$
\hat{R}_{2} \leq a_{n}^{3} E \sum_{t=1}^{n}\left|n^{-1} \sum_{s=1}^{n} \delta\left(X_{s}\right) \mathbf{K}_{h}\left(X_{t}-X_{s}\right)\right|^{3}=O_{p}\left(a_{n}^{3} n h^{-2 p}\right)=o_{p}\left(h^{-p / 2}\right)
$$

given $a_{n}=n^{-1 / 2} h^{-p / 4}$ and $h \propto n^{-\omega}$ for $\omega \in(0,1 / 2 p)$.

Finally, for the $\hat{R}_{3}$ term in (A.4), we have

$$
\begin{aligned}
\hat{R}_{3} \leq & \sum_{t=1}^{n}\left\{E\left[n^{-1} \sum_{s=1}^{n} \varepsilon_{s} \mathbf{K}_{h}\left(X_{t}-X_{s}\right)\right]^{4}\right\}^{\frac{3}{4}} \leq 4 \sum_{t=1}^{n}\left\{n^{-4} \sum_{s=1}^{n} E\left[\varepsilon_{s}^{4} \mathbf{K}_{h}^{4}\left(X_{t}-X_{s}\right)\right]\right\}^{\frac{3}{4}} \\
& +4 \sum_{t=1}^{n} n^{-4} \sum_{s_{1} \neq s_{2}}\left[E \varepsilon_{s_{1}}^{2} \varepsilon_{s_{2}}^{2} \mathbf{K}_{h}^{2}\left(X_{t}-X_{s_{1}}\right) \mathbf{K}_{h}^{2}\left(X_{t}-X_{s_{2}}\right)\right]^{\frac{3}{4}} \\
= & O_{p}\left(n^{-2} h^{-\frac{9}{4} p}\right)+O_{p}\left(n^{-1} h^{-\frac{3}{2} p}\right)=o_{p}\left(h^{-p / 2}\right) .
\end{aligned}
$$

It follows from (A.1)-(A.7) that $\hat{Q}_{n}=D \sum_{t=1}^{n} \hat{m}_{h}^{2}\left(X_{t}\right)+o_{p}\left(h^{-\frac{p}{2}}\right)$. This completes the proof.

Proof of Lemma 3.2: Because $\hat{F}_{n}(x)-F(x)=O_{p}\left(n^{-1 / 2}(\ln n)^{2}\right)$ uniformly in $x \in \mathbb{G}$ where $\hat{F}_{n}(x)$ is the empirical distribution function of $X_{t}$ (see, e.g., Bentkus, Gotse and Tikhomirov (1997)), and $n \int \hat{m}_{h}^{2}(x) f(x) d x=O_{p}\left(h^{-p}\right)$ by Theorem 3.2, we have

$$
\begin{aligned}
\sum_{t=1}^{n} \hat{m}_{h}^{2}\left(X_{t}\right) & =n \int \hat{m}_{h}^{2}(x) d F(x)+n \int \hat{m}_{h}^{2}(x) d\left[\hat{F}_{n}(x)-F(x)\right] \\
& =n \int \hat{m}_{h}^{2}(x) f(x) d x+O_{p}\left(\sup _{x \in \mathbb{G}}|\hat{F}(x)-F(x)|\right) n \int \hat{m}_{h}^{2}(x) d F(x)
\end{aligned}
$$




$$
\begin{aligned}
& =n \int \hat{m}_{h}^{2}(x) f(x) d x+O_{p}\left(n^{-1 / 2}(\ln n)^{2}\right) O_{p}\left(h^{-p}\right) \\
& =n \int \hat{m}_{h}^{2}(x) f(x) d x+o_{p}\left(h^{-p / 2}\right)
\end{aligned}
$$

given $h \propto n^{-\omega}$ for $\omega \in(0,1 / 2 p)$. This completes the proof.

Proof of Proposition 3.1: We decompose

$$
\begin{aligned}
n \int \hat{m}_{h}^{2}(x) d F(x)-n \int \tilde{m}_{h}^{2}(x) d F(x) & =n \int\left[\hat{m}_{h}^{2}(x)-\tilde{m}_{h}^{2}(x)\right] d F(x) \\
& =n \int\left[\hat{m}_{h}(x)-\tilde{m}_{h}(x)\right]^{2} d F(x)+2 n \int \tilde{m}_{h}(x)\left[\hat{m}_{h}(x)-\tilde{m}_{h}(x)\right] d F(x) \\
& =\hat{J}_{1}+2 \hat{J}_{2}, \text { say. }
\end{aligned}
$$

For $\hat{J}_{1}$, we further decompose

$$
\begin{aligned}
\hat{J}_{1}= & n \int \frac{\left[n^{-1} \sum_{s=1}^{n}\left(\hat{\varepsilon}_{s}-\varepsilon_{s}\right) \mathbf{K}_{h}\left(x-X_{s}\right)\right]^{2}}{f(x)} d x \\
& +n \int\left[n^{-1} \sum_{s=1}^{n}\left(\hat{\varepsilon}_{s}-\varepsilon_{s}\right) \mathbf{K}_{h}\left(x-X_{s}\right)\right]^{2}\left[\frac{1}{\hat{f}^{2}(x)}-\frac{1}{f^{2}(x)}\right] f(x) d x \\
= & \hat{J}_{11}+\hat{J}_{12}, \text { say. }
\end{aligned}
$$

It suffices to consider the first term $\hat{J}_{11}$ in (A.10), since the second term $\hat{J}_{12}$ is a smaller order given $\sup _{x \in \mathbb{G}} \mid \hat{f}(x)-$ $f(x) \mid \stackrel{p}{\rightarrow} 0$. Noting that $\hat{\varepsilon}_{s}-\varepsilon_{s}=\hat{\varepsilon}_{s}-\varepsilon_{n s}+\varepsilon_{n s}-\varepsilon_{s}$, we have

$$
\begin{aligned}
\hat{J}_{11}= & n \int \frac{\left[n^{-1} \sum_{s=1}^{n}\left(\hat{\varepsilon}_{s}-\varepsilon_{n s}\right)^{2} \mathbf{K}_{h}^{2}\left(x-X_{s}\right)\right]}{f(x)} d x \\
& +n \int \frac{\left[n^{-1} \sum_{s=1}^{n}\left(\varepsilon_{n s}-\varepsilon_{s}\right)^{2} \mathbf{K}_{h}^{2}\left(x-X_{s}\right)\right]}{f(x)} d x \\
& +2 n \int \frac{\left[n^{-1} \sum_{s=1}^{n}\left(\varepsilon_{n s}-\varepsilon_{s}\right)\left(\varepsilon_{n s}-\varepsilon_{s}\right) \mathbf{K}_{h}^{2}\left(x-X_{s}\right)\right]}{f(x)} d x \\
= & \hat{J}_{111}+\hat{J}_{112}+2 \hat{J}_{113},
\end{aligned}
$$

where

$$
\hat{J}_{111} \leq\left\|\hat{\theta}-\theta_{0}\right\|^{2} n \int \frac{\left[n^{-1} \sum_{s=1}^{n}\left\|\nabla_{\theta} g\left(X_{s}, \bar{\theta}\right)\right\|^{2} \mathbf{K}_{h}\left(x-X_{s}\right)\right]^{2}}{f(x)} d x=O_{p}(1)
$$

by the mean-value theorem,

$$
\begin{aligned}
\hat{J}_{112} & =n a_{n}^{2} \int \frac{\left[n^{-1} \sum_{s=1}^{n} \delta\left(X_{s}\right)^{2} \mathbf{K}_{h}^{2}\left(x-X_{s}\right)\right]}{f(x)} d x \\
& =h^{-p / 2} E\left[\delta_{n}^{2}\left(X_{t}\right)\right]\left[1+o_{p}(1)\right]
\end{aligned}
$$

and

$$
\hat{J}_{113} \leq n a_{n}\left\|\hat{\theta}-\theta_{0}\right\| \int \frac{\left[n^{-1} \sum_{s=1}^{n}\left\|\nabla_{\theta} g\left(X_{s}, \bar{\theta}\right)\left|\| \delta\left(X_{s}\right) \mathbf{K}_{h}^{2}\left(x-X_{s}\right)\right|\right]\right.}{f(x)} d x=O_{p}\left(h^{-p / 4}\right)
$$

by the mean-value theorem. It follows from (A.10)-(A.14) that

$$
\hat{J}_{1}=h^{-p / 2} E\left[\delta^{2}\left(X_{t}\right)\right]+o_{p}\left(h^{-p / 2}\right) .
$$

Next, we consider $\hat{J}_{2}$ in (A.9). Define $\tilde{m}_{h}^{*}(x)$ in the same way as $\hat{m}_{h}(x)$ with $\left\{\varepsilon_{n s}\right\}_{s=1}^{n}$ replacing $\left\{\hat{\varepsilon}_{s}\right\}_{s=1}^{n}$. 
Then, we can decompose

$$
\begin{aligned}
\hat{J}_{2} & =n \int \tilde{m}_{h}(x)\left[\hat{m}_{h}(x)-\tilde{m}_{h}^{*}(x)\right] d F(x)+n \int \tilde{m}_{h}(x)\left[\tilde{m}_{h}^{*}(x)-\tilde{m}_{h}(x)\right] d F(x) \\
& =\hat{J}_{21}+\hat{J}_{22}, \text { say. }
\end{aligned}
$$

For $\hat{J}_{21}$ in (A.16), by the second order Taylor series expansion, we have

$$
\begin{gathered}
\hat{J}_{21}=\left(\hat{\theta}_{0}-\theta_{0}\right)^{\prime} n \int \tilde{m}_{h}(x) \frac{n^{-1} \sum_{s=1}^{n} \nabla_{\theta} g\left(X_{s}, \theta_{0}\right) \mathbf{K}_{h}\left(x-X_{s}\right)}{\hat{f}(x)} d F(x) \\
+\frac{1}{2}\left(\hat{\theta}_{0}-\theta_{0}\right)^{\prime} n \int \tilde{m}_{h}(x) \frac{n^{-1} \sum_{s=1}^{n} \nabla_{\theta}^{2} g\left(X_{s}, \bar{\theta}\right) \mathbf{K}_{h}\left(x-X_{s}\right)}{\hat{f}(x)} d F(x)\left(\hat{\theta}_{0}-\theta_{0}\right) \\
=O_{p}(1)+O_{p}\left(n^{-1 / 2} h^{-p}\right)=O_{p}(1),
\end{gathered}
$$

by Hajék's projection (see Van der Vaar 1998, Chapter 11), the Cauchy-Schwarz inequality, and $h \propto n^{-\omega}$ for $\omega \in(0,1 / 2 p)$. For $\hat{J}_{22}$ in (A.16), we have

$$
\begin{aligned}
\hat{J}_{22} & =n a_{n} \int \frac{n^{-2} \sum_{t=1}^{n} \sum_{s=1}^{n} \varepsilon_{t} \delta\left(X_{s}\right) \mathbf{K}_{h}\left(x-X_{t}\right) \mathbf{K}_{h}\left(x-X_{s}\right)}{\hat{f}^{2}(x)} d F(x) \\
& =O_{p}\left(n a_{n} n^{-1 / 2}\right)=O_{p}\left(h^{-p / 4}\right) .
\end{aligned}
$$

It follows from (A.16)-(A.18) that $\hat{J}_{2}=o_{p}\left(h^{-p / 2}\right)$. This, together with (A.9) and (A.15), yields the desired result. The proof of Proposition 3.1 is completed.

Proof of Proposition 3.2: Proposition 3.2 follows from Lemmas 3.3 and 3.4 below.

Lemma 3.3: Put $\hat{H}_{q}=n^{-1} \sum_{t=2}^{n} \sum_{s=1}^{t-1} H_{n}\left(Z_{t}, Z_{s}\right)$, where $Z_{t}=\left(\varepsilon_{t}, X_{t}^{\prime}\right)^{\prime}, H_{n}\left(Z_{t}, Z_{s}\right)=2 \varepsilon_{t} \varepsilon_{s} W_{h}\left(X_{t}, X_{s}\right)$, and

$$
W_{h}\left(X_{t}, X_{s}\right)=\int \frac{\mathbf{K}_{h}\left(X_{t}-x\right) \mathbf{K}_{h}\left(X_{s}-x\right)}{f(x)} d x .
$$

Suppose Assumptions A.1 and A.4 hold, and $h \propto n^{-\omega}$ for $\omega \in(0,1 / 2 p)$. Then

$$
n \int \hat{m}_{h}^{2}(x) f(x) d x=h^{-p} \int \mathbf{K}^{2}(\mathbf{u}) d \mathbf{u} \int \sigma^{2}(x) d x+\hat{H}_{q}+o_{p}\left(h^{-p / 2}\right) .
$$

Lemma 3.4: Suppose Assumptions A.1 and A.4 hold, and $h \propto n^{-\omega}$ for $\omega \in(0,1 / 2 p)$. Define

$$
V_{q}=2 \int\left[\int \mathbf{K}(\mathbf{v}) \mathbf{K}(\mathbf{u}+\mathbf{v}) d \mathbf{v}\right]^{2} d u \int \sigma^{4}(x) d x .
$$

Then $V_{q}^{-1 / 2} h^{p / 2} \hat{H}_{q} \stackrel{d}{\longrightarrow} N(\psi, 1)$.

Proof of Lemma 3.3: Let $\hat{F}_{n}(x)$ be the empirical distribution function of $X_{t}$. We have

$$
\begin{aligned}
n \int & \tilde{m}_{h}^{2}(x) f(x) d x \\
& =n \int \frac{\left[n^{-1} \sum_{s=1}^{n} \varepsilon_{s} \mathbf{K}_{h}\left(X_{t}-X_{s}\right)\right]^{2}}{f(x)} d x+\int\left(n^{-1} \sum_{s=1}^{n} \varepsilon_{s} \mathbf{K}_{h}\left(X_{t}-X_{s}\right)\right)^{2}\left[\frac{1}{\hat{f}^{2}(x)}-\frac{1}{f^{2}(x)}\right] f(x) d x \\
& =n^{-1} \sum_{t=1}^{n} \sum_{s=1}^{n} \varepsilon_{t} \varepsilon_{s} \int \frac{\mathbf{K}_{h}\left(X_{t}-x\right) \mathbf{K}_{h}\left(X_{s}-x\right)}{f(x)} d x+O_{p}\left(n^{-1} h^{-p}\right) O_{p}\left(n^{-1 / 2} h^{-p / 2} \ln n+h^{2}\right) \\
& =n^{-1} \sum_{t=1}^{n} \varepsilon_{t}^{2} \int \frac{\mathbf{K}_{h}^{2}\left(X_{t}-x\right)}{f(x)} d x+n^{-1} \sum_{1 \leq s \leq t \leq n}^{n} 2 \varepsilon_{t} \varepsilon_{s} \int \frac{\mathbf{K}_{h}\left(X_{t}-x\right) \mathbf{K}_{h}\left(X_{s}-x\right)}{f(x)}+o_{p}\left(h^{-p / 2}\right) \\
& =\hat{C}_{q}+\hat{H}_{q}=o_{P}\left(h^{-p / 2}\right),
\end{aligned}
$$


where we have made use of the fact that $\sup _{x \in \mathbb{G}}|\hat{f}(x)-f(x)|=O_{p}\left(n^{-1 / 2} h^{-p / 2} \ln n+h^{2}\right)$ given Assumption A.2, and $h \propto n^{-\omega}$ for $\omega \in(0,1 / 2 p)$.

By change of variables, the law of iterated expectations, and Assumption A.1, we can obtain

$$
E\left(\hat{C}_{q}\right)=\iint \sigma^{2}(x) \frac{\mathbf{K}_{h}^{2}(y-x)}{f(x)} f(y) d x d y=h^{-p} \int \mathbf{K}^{2}(u) d u \int \sigma^{2}(x) d x\left[1+O\left(h^{2}\right)\right] .
$$

On the other hand, by Chebyshev's inequality, we have

$$
\hat{C}_{q}=E\left(\hat{C}_{q}\right)+O_{p}\left(n^{-1 / 2} h^{-p}\right)
$$

where we have made use of the fact that $E\left(\hat{C}_{q}-E \hat{C}_{q}\right)^{2}=O_{p}\left(n^{-1} h^{-2 p}\right)$ given Assumption A.1. Combining (A.19)-(A.21) and $p<4$ then yields the desired result. This completes the proof of Lemma 3.3.

Proof of Lemma 3.4: Because $E\left[H_{n}\left(Z_{t}, z\right)\right]=E\left[H_{n}\left(z^{\prime}, Z_{s}\right)\right]=0$ for all $z, z^{\prime}, \hat{H}_{q} \equiv n^{-1} \sum_{1 \leq s<t \leq n} H_{n}\left(Z_{t}, Z_{s}\right)$ is a degenerate $U$-statistic. Following Tenreiro's (1997) central limit theorem for degenerate $U$-statistics in a time series context, we have $\left[n^{-2} \sum_{1 \leq s<t \leq n} E\left[h^{p} H_{n}^{2}\left(Z_{t}, Z_{s}\right)\right]\right]^{-1 / 2} h^{p / 2} \hat{H}_{q} \stackrel{d}{\rightarrow} N(0,1)$ as $n \rightarrow \infty$ if the following conditions are satisfied: For some constants $\delta_{0}>0, \gamma_{0}<\frac{1}{2}$ and $\gamma_{1}>0$,

$$
\begin{aligned}
u_{n}\left(4+\delta_{0}\right) & =O\left(n^{\gamma_{0}}\right) ; \\
v_{n}(2) & =o(1) ; \\
w_{n}\left(2+\frac{\delta_{0}}{2}\right) & =o\left(n^{\frac{1}{2}}\right) ; \\
z_{n}(2) n^{\gamma_{1}} & =O(1),
\end{aligned}
$$

where

$$
\begin{aligned}
u_{n}(r) & =h^{p / 2} \max \left\{\max _{1 \leq t \leq n}\left\|H_{n}\left(Z_{t}, Z_{0}\right)\right\|_{r},\left\|H_{n}\left(Z_{0}, \bar{Z}_{0}\right)\right\|_{r}\right\}, \\
v_{n}(r) & =h^{p} \max \left\{\max _{1 \leq t \leq n}\left\|G_{n 0}\left(Z_{t}, Z_{0}\right)\right\|_{r},\left\|G_{n 0}\left(Z_{0}, \bar{Z}_{0}\right)\right\|_{r}\right\}, \\
w_{n}(r) & =h^{p}\left\|G_{n 0}\left(Z_{0}, Z_{0}\right)\right\|_{r} \\
z_{n}(r) & =h^{p} \max _{0 \leq t \leq n, 1 \leq s \leq n} \max \left\{\left\|G_{n s}\left(Z_{t}, Z_{0}\right)\right\|_{r},\left\|G_{n s}\left(Z_{0}, Z_{t}\right)\right\|_{r},\left\|G_{n s}\left(Z_{0}, \bar{Z}_{0}\right)\right\|_{r}\right\},
\end{aligned}
$$

$G_{n s}(u, v)=E\left[H_{n}\left(Z_{s}, u\right) H_{n}\left(Z_{0}, v\right)\right]$ for $s \in \mathbb{N}$ and $u, v \in \mathbb{R}^{p}, \bar{Z}_{0}$ is an independent copy of $Z_{0}$, and $\|\xi\|_{r}=E^{\frac{1}{r}}|\xi|^{r}$.

We first show $n^{-2} \sum_{1 \leq s<t \leq n} h^{p} E\left[H_{n}^{2}\left(Z_{t}, Z_{s}\right)\right] \rightarrow V$ as $n \rightarrow \infty$. By change of variables and Assumption A.1, it is straightforward to calculate that

$$
\begin{aligned}
n^{-2} \sum_{1 \leq s<t \leq n} h^{p} E\left[H_{n}^{2}\left(Z_{t}, Z_{s}\right)\right] & =4 h^{p} n^{-2} \sum_{1 \leq s<t \leq n} E\left[\varepsilon_{t}^{2} \varepsilon_{s}^{2} W_{h}^{2}\left(X_{t}, X_{s}\right)\right] \\
& \rightarrow 2 \int\left[\int \mathbf{K}(v) \mathbf{K}(u+v) d v\right]^{2} d u \int \sigma^{4}(x) d x \equiv V_{q} .
\end{aligned}
$$

We now verify Conditions (A.22)-(A.25). First, we shall show Condition (A.22): $u_{n}\left(4+\delta_{0}\right)=O\left(n^{\gamma_{0}}\right)$ for some $\delta_{0}>0, \gamma_{0}<\frac{1}{2}$. By the Cauchy-Schwarz inequality and change of variables, we have for all $t \geq 0$,

$$
\begin{aligned}
E\left|h^{p / 2} H_{n}\left(Z_{t}, Z_{0}\right)\right|^{r} & \leq 2^{\gamma} h^{\frac{p}{2} r}\left(E\left|\varepsilon_{t} \varepsilon_{0}\right|^{c r}\right)^{\frac{1}{c}}\left(E\left|W_{h}\left(X_{t}, X_{0}\right)\right|^{c r}\right)^{\frac{1}{c}} \\
& \leq C h^{\frac{p}{2} r}\left[\int\left|W_{h}\left(x, x_{0}\right)\right|^{c r} f_{X_{t}, X_{0}}\left(x, x_{0}\right) d x d x_{0}\right]^{\frac{1}{c}} \\
& \leq C h^{\frac{p}{2} r}\left(h^{-p c r} h^{p}\right)^{\frac{1}{c}} \leq C h^{-\frac{r}{2} p+\frac{p}{c}}
\end{aligned}
$$


for all $c>1$, and given $E\left(\varepsilon_{t}^{8+\delta}\right) \leq C$, where $f_{X_{t}, X_{0}}\left(x, x_{0}\right)$ is the joint probability density of $\left(X_{t}, X_{0}\right)$. We then obtain $\left\|h^{p / 2} H_{n}\left(Z_{t}, Z_{0}\right)\right\|_{r}=\left(C h^{-\frac{r}{2} p+\frac{p}{c}}\right)^{\frac{1}{r}} \leq C h^{-\frac{p}{2}+\frac{p}{c r}}$. Given $h \propto n^{-\omega}$ for $\omega \in(0,1 / 2 p)$, we have

$$
\left\|h^{p / 2} H_{n}\left(Z_{t}, Z_{0}\right)\right\|_{r} \leq C n^{\omega p\left(\frac{1}{2}-\frac{2}{8+\delta}\right)},
$$

with $c=\frac{8+\delta}{2 r}$ and if $r<4+\frac{\delta}{2}$. By a similar argument and replacing $f_{X_{t}, X_{0}}\left(x, x_{0}\right)$ with $f(x) f\left(x_{0}\right)$, we can obtain the same order of magnitude for $\left\|h^{p / 2} H_{n}\left(Z_{0}, \bar{Z}_{0}\right)\right\|_{r}$. Hence, we obtain

$$
u_{n}(r) \leq C n^{\omega p\left(\frac{1}{2}-\frac{2}{8+\delta}\right)},
$$

and Condition (A.22) holds by setting $\gamma_{0}=\omega p\left(\frac{1}{2}-\frac{2}{8+\delta}\right)$.

Now we verify Condition (A.23). Note that for all $s \geq 0$, we have

$$
G_{n s}\left(z, z^{\prime}\right)=4 \varepsilon \varepsilon^{\prime} E\left[\varepsilon_{s} \varepsilon_{0} W_{h}\left(X_{s}, x\right) W_{h}\left(X_{0}, x^{\prime}\right)\right],
$$

where $z=(\varepsilon, x)$ and $z^{\prime}=\left(\varepsilon^{\prime}, x^{\prime}\right)$. To compute the order of magnitude for $v_{n}(r)$, we first consider the case of $s=0$. We have

$$
G_{n 0}\left(z, z^{\prime}\right)=4 \varepsilon \varepsilon^{\prime} E_{0}\left[\sigma^{2}\left(\bar{X}_{0}\right) W_{h}\left(\bar{X}_{0}, x\right) W_{h}\left(\bar{X}_{0}, x^{\prime}\right)\right]
$$

where $E_{0}(\cdot)$ is an expectation taken over $\left(\bar{X}_{0}, \bar{\varepsilon}_{0}\right)$. By the Cauchy-Schawarz inequality and change of variables, we have

$$
\begin{aligned}
E\left|h^{p} G_{n 0}\left(Z_{t}, Z_{0}\right)\right|^{2} & \leq 16 h^{2 p} E\left|\varepsilon_{t}^{2} \varepsilon_{0}^{2}\left[E_{0} \sigma^{2 c}\left(\bar{X}_{0}\right)\right]^{\frac{2}{c}}\left[E_{0} W_{h}^{c}\left(\bar{X}_{0}, X_{t}\right) W_{h}^{c}\left(\bar{X}_{0}, X_{0}\right)\right]^{\frac{2}{c}}\right| \\
& \leq 16 h^{2 p} C\left[E\left|\varepsilon_{t}^{4} \varepsilon_{0}^{4}\right|\right]^{\frac{1}{2}}\left\{E\left|E_{0}\left(W_{h}^{c}\left(\bar{X}_{0}, X_{t}\right) W_{h}^{c}\left(\bar{X}_{0}, X_{0}\right)\right)\right|^{\frac{4}{c}}\right\}^{\frac{1}{2}} \\
& \leq C h^{2 p}\left\{E\left|h^{-2 c p+p} \mathbf{A}_{c, h}\left(X_{t}, X_{0}\right)\right|^{\frac{4}{c}}\right\}^{\frac{1}{2}}=O\left(h^{\left(\frac{2}{c}-\frac{3}{2}\right) p}\right),
\end{aligned}
$$

for any $c>1$, where

$$
E_{0}\left[W_{h}^{c}\left(\bar{X}_{0}, X_{t}\right) W_{h}^{c}\left(\bar{X}_{0}, X_{0}\right)\right]=h^{-2 c p+p} \mathbf{A}_{c, h}\left(X_{t}, X_{0}\right)
$$

by change of variables, where $\mathbf{A}_{c, h}\left(X_{t}, X_{0}\right)$ is a function similar to $\mathbf{K}_{h}\left(X_{t}-X_{0}\right)$. Thus, we obtain $\left\|h^{p} G_{n 0}\left(Z_{t}, Z_{0}\right)\right\|_{2} \leq$ $C h^{\left(\frac{1}{c}-\frac{3}{4}\right) p}$. By a similar argument, we can obtain the same order for $\left\|h^{p} G_{n 0}\left(Z_{t}, \bar{Z}_{0}\right)\right\|_{2}$. Thus, we have

$$
v_{n}(r) \leq C h^{\left(\frac{1}{c}-\frac{3}{4}\right) p},
$$

and Condition (A.23) holds, i.e., $v_{n}(2)=o(1)$, with $1<c<\frac{4}{3}$.

Next, to verify Condition (A.24), we shall evaluate $\left\|h^{p} G_{n 0}\left(Z_{0}, \bar{Z}_{0}\right)\right\|_{r}$ for $r<2+\frac{\delta_{0}}{4}$. By the Cauchy-Schwarz inequality and change of variables, we have

$$
\begin{aligned}
E\left|h^{p} G_{n 0}\left(Z_{0}, Z_{0}\right)\right|^{r} & \leq 4^{\gamma} h^{2 p} E\left|\varepsilon_{0}^{2 r} \sigma^{2 c}\left(\bar{X}_{0}\right)^{\frac{r}{c}}\left[E_{0} W_{h}^{2 c}\left(\bar{X}_{0}, X_{0}\right)\right]^{\frac{r}{c}}\right| \\
& \leq C h^{r p}\left(E \varepsilon_{0}^{4 r}\right)^{\frac{1}{2}}\left[E\left|E_{0} W_{h}^{2 c}\left(\bar{X}_{0}, X_{0}\right)\right|^{\frac{2 r}{c}}\right]^{\frac{1}{2}}=O\left(h^{r p\left(\frac{1}{c}-1\right)}\right),
\end{aligned}
$$

where $E_{0}\left[W_{h}^{2}\left(\bar{X}_{0}, X_{0}\right)\right]=\int W_{h}^{2 c}\left(\bar{x}_{0}, X_{0}\right) f_{\bar{X}_{0}}\left(\bar{x}_{0}\right) d \bar{x}_{0}=O\left(h^{(1-2 c) p}\right)$ by change of variables. As a result, we obtain $\left\|h^{p} G_{n 0}\left(Z_{0}, Z_{0}\right)\right\|_{r} \leq C h^{p\left(\frac{1}{c}-1\right)}=C n^{\omega p\left(1-\frac{1}{c}\right)}$ given $h \propto n^{-\omega}$. Thus, Condition (A.24) holds by choosing $c$ sufficiently small subject to the constraint of $c>1$.

Finally, we verify Condition (A.25). We first consider the case $t=0$ and $s \neq 0$. We have, by the Cauchy- 
Schwarz inequality and change of variables,

$$
\begin{aligned}
E\left|h^{p} G_{n s}\left(Z_{0}, Z_{0}\right)\right|^{2} & \leq 16 h^{2 p} E\left|\varepsilon_{0}^{4}\left(E_{0} \bar{\varepsilon}_{s}^{c} \bar{\varepsilon}_{0}^{c}\right)^{\frac{2}{c}}\left[E_{0} W_{h}^{c}\left(\bar{X}_{s}, X_{0}\right) W_{h}^{c}\left(\bar{X}_{0}, X_{0}\right)\right]^{\frac{2}{c}}\right| \\
& \leq 16 h^{2 p}\left(E\left|\varepsilon_{0}\right|^{8}\right)^{\frac{1}{2}}\left[E\left|E_{0}^{\frac{4}{c}} W_{h}^{c}\left(\bar{X}_{s}, X_{0}\right) W_{h}^{c}\left(\bar{X}_{0}, X_{0}\right)\right|\right]^{\frac{1}{2}}=O\left(h^{2\left(\frac{2}{c}-1\right) p}\right),
\end{aligned}
$$

where $E_{0}\left[W_{h}^{c}\left(\bar{X}_{s}, X_{0}\right) W_{h}^{c}\left(\bar{X}_{0}, X_{0}\right)\right]=\int W_{h}^{c}\left(\bar{x}, X_{0}\right) W_{h}^{c}\left(\bar{x}_{0}, X_{0}\right) f_{\bar{X}_{s} \bar{X}_{0}}\left(\bar{x}, \bar{x}_{0}\right) d \bar{x} d \bar{x}_{0}=O\left(h^{2(1-c) p}\right)$ by change of variables. Thus, we obtain $\left\|h^{p} G_{n s}\left(Z_{0}, Z_{0}\right)\right\|_{2} \leq\left[C h^{2\left(\frac{2}{c}-1\right) p}\right]^{\frac{1}{2}}=C h^{\left(\frac{2}{c}-1\right) p}$, and thus we have $n^{\gamma_{1}}\left\|h^{p} G_{n s}\left(Z_{0}, Z_{0}\right)\right\|_{2}=$ $n^{\left(1-\frac{2}{c}\right) \phi p+\gamma_{1}}$, if $h=O\left(n^{-\phi}\right)$. Therefore, we obtain $\left\|h^{p} G_{n s}\left(Z_{0}, Z_{0}\right)\right\|_{2}=O\left(n^{-\gamma_{1}}\right)$ with $\gamma_{1}=\left(\frac{c}{2}-1\right) \phi p$, if we choose $c$ small enough for $1<c<2$. For the case $t \neq 0$ and $s \neq 0$, by a similar argument, we have $\left\|h^{p} G_{n s}\left(Z_{t}, Z_{0}\right)\right\|_{2} \leq\left[C h^{2\left(\frac{2}{c}-1\right) p}\right]^{\frac{1}{2}}=O\left(h^{\left(\frac{2}{c}-1\right) p}\right)$. Thus, Condition (A.25) holds with $\gamma_{1}=\left(\frac{c}{2}-1\right) \omega p$, provided we choose $c$ small enough with $1<c<2$. Therefore, Conditions (A.22)-(A.25) hold, and we have $V_{q}^{-1 / 2} h^{p / 2} \hat{H}_{q} \stackrel{d}{\longrightarrow} N(0,1)$ by Tenreiro's (1997) central limit theorem. This proves Lemma 3.4.

Proof of Theorem 3(ii): We shall now derive the asymptotic distribution of $\lambda_{n}$ under $\mathbb{H}_{n}\left(a_{n}\right)$. From Lemmas 3.5 and 3.6 and Propositions 3.3 and 3.4 below, we have

$$
\left[\lambda_{n}-h^{-p} \sigma^{-2} c(K) \int \sigma^{2}(x) d x\right] / \sqrt{2 \sigma^{-4} d(K) \int \sigma^{4}(x) d x} \stackrel{d}{\longrightarrow} N(\xi, 1)
$$

under $\mathbb{H}_{n}\left(a_{n}\right)$ with $a_{n}=n^{-1 / 2} h^{-4 / 9}$.

Lemma 3.5: Under the conditions of Theorem 3, $\lambda_{n}=\frac{n}{2} \frac{S S R_{0}-S S R_{1}}{S S R_{1}}+o_{p}\left(h^{-p / 2}\right)$ under $\mathbb{H}_{n}\left(a_{n}\right)$ with $a_{n}=$ $n^{-1 / 2} h^{-p / 4}$.

Lemma 3.6: Under the conditions of Theorem 3, $\hat{\sigma}_{n}^{2} \equiv n^{-1} S S R_{1}=\sigma^{2}+O_{p}\left(n^{-1 / 2}\right)$ under $\mathbb{H}_{n}\left(a_{n}\right)$ with $a_{n}=n^{-1 / 2} h^{-p / 4}$.

Proposition 3.3: Let $\widetilde{S S R_{0}}$ and $\widetilde{S S R_{1}}$ be defined in the same way as $S S R_{0}$ and $S S R_{1}$ respectively, with $\left\{\varepsilon_{t}\right\}_{t=1}^{n}$ replacing $\left\{\hat{\varepsilon}_{t}=Y_{t}-m_{h}\left(X_{t}, \theta_{0}\right)\right\}_{t=1}^{n}$. Then under the conditions of Theorem 3, SSR $-S S R_{1}=\widetilde{S S R}_{0}-\widetilde{S S R}_{1}+$ $h^{-p / 2} E\left[\delta^{2}\left(X_{t}\right)\right]+o_{p}\left(h^{-p / 2}\right)$ under $\mathbb{H}_{n}\left(a_{n}\right)$ with $a_{n}=n^{-1 / 2} h^{-p / 4}$.

Proposition 3.4: Under the conditions of Theorem 3 and $\mathbb{H}_{n}\left(a_{n}\right)$ with $a_{n}=n^{-1 / 2} h^{-p / 4}$,

$$
\left[\frac{\widetilde{S S R}_{0}-\widetilde{S S R}_{1}}{2 \sigma^{2}}-h^{-p} \sigma^{-2} c(K) \int \sigma^{2}(x) d x\right] / \sqrt{2 \sigma^{-4} d(K) \int \sigma^{4}(x) d x} \stackrel{d}{\longrightarrow} N(\xi, 1) .
$$

Proof of Lemma 3.5: Given the inequality that $|\ln (1+z)-z| \leq|z|^{2}$ for small $z$, we have

$$
\begin{aligned}
\lambda_{n} & =\frac{n}{2} \frac{S S R_{0}-S S R_{1}}{S S R_{1}}+O_{p}\left[\frac{n}{2}\left(\frac{S S R_{0}-S S R_{1}}{S S R_{1}}\right)^{2}\right] \\
& =\frac{n}{2} \frac{S S R_{0}-S S R_{1}}{S S R_{1}}+O_{p}\left(n^{-1} h^{-2 p}\right)=\frac{n}{2} \frac{S S R_{0}-S S R_{1}}{S S R_{1}}+o_{p}\left(h^{-p / 2}\right),
\end{aligned}
$$

where we have used the facts that $n^{-1} S S R_{1} \stackrel{p}{\rightarrow} \sigma^{2}$ under $\mathbb{H}_{n}\left(a_{n}\right)$ by Lemma 3.6 and $S S R_{0}-S S R_{1}=O_{P}\left(h^{-p}\right)$ by Propositions 3.3 and 3.4, as well as $n h^{3 p / 2} \rightarrow \infty$.

Proof of Lemma 3.6: Put $\hat{A}_{t}=\hat{\varepsilon}_{t}-\varepsilon_{t}$ and $\hat{B}_{t}=\hat{m}_{h}\left(X_{t}\right)-\tilde{m}_{h}\left(X_{t}\right)$, where $\hat{m}_{h}\left(X_{t}\right)$ is given in (4.1) and $\tilde{m}_{h}\left(X_{t}\right)$ is defined in the same way as $\hat{m}_{h}\left(X_{t}\right)$ with $\varepsilon_{t}$ replacing $\hat{\varepsilon}_{t}$. Then we have

$$
n^{-1} S S R_{1}=n^{-1} \sum_{t=1}^{n}\left[\hat{\varepsilon}_{t}-\hat{m}_{h}\left(X_{t}\right)\right]^{2}=n^{-1} \sum_{t=1}^{n}\left\{\left(\hat{\varepsilon}_{t}-\varepsilon_{t}\right)-\left[\hat{m}_{h}\left(X_{t}\right)-\tilde{m}_{h}\left(X_{t}\right)\right]+\left[\varepsilon_{t}-\tilde{m}_{h}\left(X_{t}\right)\right]\right\}^{2}
$$


and we can write

$$
n^{-1} S S R_{1}=n^{-1} \widetilde{S S R_{1}}+n^{-1} \sum_{t=1}^{n}\left(\hat{A}_{t}-\hat{B}_{t}\right)^{2}+2 n^{-1} \sum_{t=1}^{n}\left[\varepsilon_{t}-\tilde{m}_{h}\left(X_{t}\right)\right]\left(\hat{A}_{t}-\hat{B}_{t}\right),
$$

where $\widetilde{S S R}_{1}=\sum_{t=1}^{n}\left[\varepsilon_{t}-\tilde{m}_{h}\left(X_{t}\right)\right]^{2}$.

For the second term in (A.27), recalling $w_{s t}=\frac{\mathbf{K}_{h}\left(X_{t}-X_{s}\right)}{\sum_{t=1}^{n} \mathbf{K}_{h}\left(X_{t}-X_{s}\right)}$, and $\sum_{t=1}^{n} w_{s t}=1$ for all $s$, we have $\hat{B}_{t}=$ $\sum_{s=1}^{n} w_{s t} \hat{A}_{s}$. Under $\mathbb{H}_{n}\left(a_{n}\right)$, we have

$$
\hat{A}_{t}=Y_{t}-g\left(X_{t}, \hat{\theta}_{0}\right)-\varepsilon_{t}=g\left(X_{t}, \theta_{0}\right)-g\left(X_{t}, \hat{\theta}_{0}\right)+a_{n} \delta\left(X_{t}\right) .
$$

It follows that

$$
\begin{gathered}
n^{-1} \sum_{t=1}^{n}\left(\hat{A}_{t}-\hat{B}_{t}\right)^{2} \leq 8 n^{-1} \sum_{t=1}^{n}\left[g\left(X_{t}, \theta_{0}\right)-g\left(X_{t}, \hat{\theta}_{0}\right)\right]^{2}+8 n^{-1} a_{n}^{2} \sum_{t=1}^{n} \delta^{2}\left(X_{t}\right) \\
=O_{p}\left(n^{-1}\right)+O_{p}\left(a_{n}^{2}\right)=O_{p}\left(n^{-1} h^{-p / 2}\right)=o_{p}\left(n^{-1 / 2}\right)
\end{gathered}
$$

where $a_{n}=n^{-1 / 2} h^{-p / 4}$, and the first term is $O_{p}\left(n^{-1}\right)$ by the mean-value theorem, and Assumptions A.2 and A.3. For the third term in (A.27), we have

$$
n^{-1} \sum_{t=1}^{n}\left[\varepsilon_{t}-\tilde{m}_{h}\left(X_{t}\right)\right]\left(\hat{A}_{t}-\hat{B}_{t}\right)=n^{-1} \sum_{t=1}^{n} \varepsilon_{t}\left(\hat{A}_{t}-\hat{B}_{t}\right)-n^{-1} \sum_{t=1}^{n} \tilde{m}_{h}\left(X_{t}\right)\left(\hat{A}_{t}-\hat{B}_{t}\right)=\hat{T}_{1}-\hat{T}_{2} .
$$

For the $\hat{T}_{2}$ term, by the Cauchy-Schwarz inequality, we have

$$
\begin{aligned}
\left|\hat{T}_{2}\right| & \leq\left[n^{-1} \sum_{t=1}^{n} \tilde{m}_{h}^{2}\left(X_{t}\right)\right]^{\frac{1}{2}}\left[n^{-1} \sum_{t=1}^{n}\left(\hat{A}_{t}-\hat{B}_{t}\right)^{2}\right]^{\frac{1}{2}} \\
& =O_{p}\left(n^{-1 / 2} h^{-p / 2}\right) O_{p}\left(n^{-1 / 2} h^{-p / 4}\right)=o_{p}\left(n^{-1 / 2}\right),
\end{aligned}
$$

given $n h^{3 p / 2} \rightarrow \infty$ and (A.29), where $n^{-1} \sum_{t=1}^{n} \tilde{m}_{h}^{2}\left(X_{t}\right)=O_{p}\left(n^{-1} h^{-p}\right)$ by Markov's inequality, $E\left(\varepsilon_{t} \mid X_{t}\right)=0$ a.s. and Assumption A.1.

For the $\hat{T}_{1}$ term, we decompose

$$
\hat{T}_{1}=n^{-1} \sum_{t=1}^{n} \varepsilon_{t} \hat{A}_{t}-n^{-1} \sum_{t=1}^{n} \varepsilon_{t} \hat{B}_{t}=\hat{T}_{11}-\hat{T}_{12}, \text { say. }
$$

Here, using (A.29), we have

$$
\begin{aligned}
\hat{T}_{11} & =n^{-1} \sum_{t=1}^{n} \varepsilon_{t}\left[g\left(X_{t}, \theta_{0}\right)-g\left(X_{t}, \hat{\theta}_{0}\right)\right]+a_{n} n^{-1} \sum_{t=1}^{n} \varepsilon_{t} \delta\left(X_{t}\right) \\
& =O_{p}\left(n^{-1}\right)+O_{p}\left(n^{-1 / 2} a_{n}\right)=o_{p}\left(n^{-1 / 2}\right)
\end{aligned}
$$

where the first term is $O_{p}\left(n^{-1}\right)$ by a second order Taylor series expansion, Chebyshev's inequality, the CauchySchwarz inequality, and Assumptions A.2 and A.3; the second term is $O_{p}\left(n^{-1} h^{-p / 4}\right)$ by $a_{n}=n^{-1 / 2} h^{-p / 4}$, $E\left[\delta^{4}\left(X_{t}\right)\right] \leq C$ and Chebyshev's inequality.

For the $\hat{T}_{12}$ term in (A.32), recalling $\hat{B}_{t}=\sum_{s=1}^{n} w_{s t} \hat{A}_{s}$ and using (A.28), we have

$$
\begin{aligned}
\hat{T}_{12} & =n^{-1} \sum_{t=1}^{n} \varepsilon_{t}\left\{\sum_{s=1}^{n} w_{s t}\left[g\left(X_{s}, \theta_{0}\right)-g\left(X_{s}, \hat{\theta}_{0}\right)\right]\right\}+a_{n} n^{-1} \sum_{t=1}^{n} \varepsilon_{t} \sum_{s=1}^{n} w_{s t} \delta\left(X_{s}\right) \\
& =O_{p}\left(n^{-1}\right)+O_{p}\left(n^{-1 / 2} a_{n}\right)=o_{p}\left(n^{-1 / 2}\right)
\end{aligned}
$$

where the order of each term follows by a similar reasoning to that for the $\hat{T}_{11}$ term. 
Finally, for the first term $n^{-1} \widetilde{S S R}_{1}$ in (A.27), we have

$$
\begin{aligned}
n^{-1} \widetilde{S S R}_{1} & =n^{-1} \sum_{t=1}^{n} \varepsilon_{t}^{2}-n^{-1} 2 \sum_{t=1}^{n} \varepsilon_{t} \tilde{m}_{h}\left(X_{t}\right)+n^{-1} \sum_{t=1}^{n} \tilde{m}_{h}^{2}\left(X_{t}\right) \\
& =\left[\sigma^{2}+O_{p}\left(n^{-1 / 2}\right)\right]+O_{p}\left(n^{-1} h^{-p / 2}\right)+O_{p}\left(n^{-1} h^{-p}\right)=\sigma^{2}+O_{p}\left(n^{-1 / 2}\right),
\end{aligned}
$$

given $\sum_{t=1}^{n} \tilde{m}_{h}^{2}\left(X_{t}\right)=O_{p}\left(n^{-1} h^{-p}\right)$ by Markov's inequality and $\sum_{t=1}^{n} \varepsilon_{t} \tilde{m}_{h}\left(X_{t}\right)=O_{p}\left(n^{-1} h^{-p}\right)$. Collecting (A.27) and (A.29)-(A.35) yields the desired result of this lemma.

Proof of Proposition 3.3: We first write

$$
S S R_{0}-S S R_{1}=\left[\widetilde{S S R}_{0}-\widetilde{S S R}_{1}\right]+\left[S S R_{0}-\widetilde{S S R}_{0}\right]-\left[S S R_{1}-\widetilde{S S R}_{1}\right]
$$

For the second term in (A.36), we have

$$
S S R_{0}-\widetilde{S S R}_{0}=\sum_{t=1}^{n} \hat{\varepsilon}_{t}^{2}-\sum_{t=1}^{n} \varepsilon_{t}^{2}=\sum_{t=1}^{n} \hat{A}_{t}^{2}+2 \sum_{t=1}^{n} \varepsilon_{t} \hat{A}_{t}
$$

where $\hat{A}_{t}=\hat{\varepsilon}_{t}-\varepsilon_{t}$ as in the proof of Lemma 3.6. For the first term in (A.37), using (A.28), we have

$$
\begin{aligned}
\sum_{t=1}^{n} \hat{A}_{t}^{2} & =\sum_{t=1}^{n}\left[g\left(X_{t}, \theta_{0}\right)-g\left(X_{t}, \hat{\theta}_{0}\right)\right]^{2}+2 a_{n} \sum_{t=1}^{n}\left[g\left(X_{t}, \theta_{0}\right)-g\left(X_{t}, \hat{\theta}_{0}\right)\right] \delta\left(X_{t}\right)+a_{n}^{2} \sum_{t=1}^{n} \delta^{2}\left(X_{t}\right) \\
& =O_{p}(1)+O_{p}\left(a_{n} n^{1 / 2}\right)+n a_{n}^{2} E\left[\delta^{2}\left(X_{t}\right)\right]+O_{p}\left(n^{1 / 2} a_{n}^{2}\right) \\
& =h^{-p / 2} E\left[\delta^{2}\left(X_{t}\right)\right]+o_{p}\left(h^{-p / 2}\right),
\end{aligned}
$$

where $n^{-1} \sum_{t=1}^{n} \delta^{2}\left(X_{t}\right)=E \delta^{2}\left(X_{t}\right)+O_{p}\left(n^{-1 / 2}\right)$ by Chebyshev's inequality and Assumption A.1.

For the second term in (A.37), by using (A.28) and a second order Taylor series expansion, we have

$$
\begin{aligned}
\sum_{t=1}^{n} \varepsilon_{t} \hat{A}_{t} & =\left[\sum_{t=1}^{n} \varepsilon_{t} \nabla_{\theta} g\left(X_{t}, \theta_{0}\right)\right]\left(\hat{\theta}-\theta_{0}\right)+\frac{1}{2}\left(\hat{\theta}-\theta_{0}\right)^{\prime}\left[\sum_{t=1}^{n} \varepsilon_{t} \nabla_{\theta}^{2} g\left(X_{t}, \bar{\theta}\right)\right]\left(\hat{\theta}-\theta_{0}\right)+a_{n} \sum_{t=1}^{n} \varepsilon_{t} \delta\left(X_{t}\right) \\
& =O_{p}(1)+O_{p}(1)+O_{p}\left(n^{1 / 2} a_{n}\right)
\end{aligned}
$$

given $E \delta^{2}\left(X_{t}\right) \leq C$ and Assumptions A.1-A.3.

Now, we consider the third term in (A.36). We decompose

$$
\begin{aligned}
S S R_{1}-\widetilde{S S R}_{1}= & \sum_{t=1}^{n}\left[\left(\hat{\varepsilon}_{t}-\hat{m}_{h}\left(X_{t}\right)\right)-\left(\varepsilon_{t}-\tilde{m}_{h}\left(X_{t}\right)\right)\right]^{2} \\
& +2 \sum_{t=1}^{n}\left[\varepsilon_{t}-\tilde{m}_{h}\left(X_{t}\right)\right]\left\{\left[\hat{\varepsilon}_{t}-\hat{m}_{h}\left(X_{t}\right)\right]-\left[\varepsilon_{t}-\tilde{m}_{h}\left(X_{t}\right)\right]\right\} \\
= & \hat{M}_{1}+2 \hat{M}_{2}, \text { say. }
\end{aligned}
$$

We first consider $\hat{M}_{1}$ in (A.40). Recalling $\tilde{m}_{h}^{*}\left(X_{t}\right)=\sum_{s=1}^{n} w_{t s} \varepsilon_{n s}$, as defined in the proof of Proposition of 3.1, we can write

$$
\begin{aligned}
\hat{M}_{1} & =\sum_{t=1}^{n}\left[\hat{\varepsilon}_{t}-\varepsilon_{n t}+\varepsilon_{n t}-\varepsilon_{t}-\left(\hat{m}_{h}\left(X_{t}\right)-\tilde{m}_{h}^{*}\left(X_{t}\right)\right)-\left(\tilde{m}_{h}^{*}\left(X_{t}\right)-\tilde{m}_{h}\left(X_{t}\right)\right)\right]^{2} \\
& \leq 2 \sum_{t=1}^{n}\left[\left(\hat{\varepsilon}_{t}-\varepsilon_{n t}\right)-\left(\hat{m}_{h}\left(X_{t}\right)-\tilde{m}_{h}^{*}\left(X_{t}\right)\right)\right]^{2}+2 \sum_{t=1}^{n}\left[\left(\varepsilon_{n t}-\varepsilon_{t}\right)-\left(\tilde{m}_{h}^{*}\left(X_{t}\right)-\tilde{m}_{h}\left(X_{t}\right)\right)\right]^{2} \\
& =2 \hat{M}_{11}+2 \hat{M}_{12}, \text { say. }
\end{aligned}
$$


For the $\hat{M}_{11}$ term in (A.41), we have

$$
\begin{aligned}
\hat{M}_{11} & \leq 2 \sum_{t=1}^{n}\left(\hat{\varepsilon}_{t}-\varepsilon_{n t}\right)^{2}+2 \sum_{s=1}^{n}\left(\hat{\varepsilon}_{s}-\varepsilon_{n s}\right)^{2}\left(\sum_{t=1}^{n} \sum_{s=1}^{n} w_{t s}\right) \\
& \leq 4 \sum_{t=1}^{n}\left(\hat{\varepsilon}_{t}-\varepsilon_{n t}\right)^{2}=4 \sum_{t=1}^{n}\left[m_{h}\left(X_{t}, \theta_{0}\right)-m_{h}\left(X_{t}, \hat{\theta}_{0}\right)\right]^{2}=O_{p}(1),
\end{aligned}
$$

by the mean value theorem and Assumptions A.1-A.3.

For the $\hat{M}_{12}$ term in (A.41), we have

$$
\begin{aligned}
\hat{M}_{12} & =a_{n}^{2} \sum_{t=1}^{n}\left[\delta\left(X_{t}\right)-\sum_{s=1}^{n} \tilde{w}_{t s} \delta\left(X_{s}\right)+\sum_{s=1}^{n}\left(\tilde{w}_{t s}-w_{t s}\right) \delta\left(X_{s}\right)\right]^{2} \\
& =o_{p}\left(a_{n}^{2} n\right)+O_{p}\left(a_{n}^{2} h^{-p}\right)+o_{p}\left(a_{n}^{2} n\right)
\end{aligned}
$$

given the condition that $\delta(\cdot)$ is continuous with $E\left[\delta^{2}\left(X_{t}\right)\right] \leq C$, and the Assumption A.1.

Next, we consider the $\hat{M}_{2}$ term in (A.40). We decompose

$$
\begin{aligned}
\hat{M}_{2}= & \sum_{t=1}^{n}\left[\varepsilon_{t}-\tilde{m}_{h}\left(X_{t}\right)\right]\left(\hat{\varepsilon}_{t}-\varepsilon_{n t}\right)+\sum_{t=1}^{n}\left[\varepsilon_{t}-\tilde{m}_{h}\left(X_{t}\right)\right]\left(\varepsilon_{n t}-\varepsilon_{t}\right) \\
& -\sum_{t=1}^{n}\left[\varepsilon_{t}-\tilde{m}_{h}\left(X_{t}\right)\right]\left[\hat{m}_{h}\left(X_{t}\right)-\tilde{m}_{h}^{*}\left(X_{t}\right)\right]-\sum_{t=1}^{n}\left[\varepsilon_{t}-\tilde{m}_{h}\left(X_{t}\right)\right]\left[\tilde{m}_{h}^{*}\left(X_{t}\right)-\tilde{m}_{h}\left(X_{t}\right)\right] \\
= & \hat{M}_{21}+\hat{M}_{22}-\hat{M}_{23}-\hat{M}_{24}, \text { say. }
\end{aligned}
$$

For the $\hat{M}_{21}$ term in (A.44), by the second order Taylor series expansion, Chebyshev's inequality and the CauchySchwarz inequality, we have

$$
\begin{aligned}
\hat{M}_{21} & =\sum_{t=1}^{n} \varepsilon_{t}\left[g\left(X_{t}, \theta_{0}\right)-g\left(X_{t}, \hat{\theta}_{0}\right)\right]-\sum_{t=1}^{n} \tilde{m}_{h}\left(X_{t}\right)\left[g\left(X_{t}, \theta_{0}\right)-g\left(X_{t}, \hat{\theta}_{0}\right)\right] \\
& =O_{p}(1)+O_{p}(1)+O_{p}\left(n^{-1 / 2} h^{-p / 2}\right) .
\end{aligned}
$$

For the $\hat{M}_{22}$ term in (A.44), we have

$$
\hat{M}_{22}=a_{n} \sum_{t=1}^{n} \varepsilon_{t} \delta\left(X_{t}\right)-a_{n} \sum_{t=1}^{n} \tilde{m}_{h}\left(X_{t}\right) \delta\left(X_{t}\right)=O_{p}\left(n^{1 / 2} a_{n}\right)+O_{p}\left(n^{1 / 2} a_{n}\right),
$$

where $n^{-1 / 2} \sum_{t=1}^{n} \tilde{m}_{h}\left(X_{t}\right) \delta\left(X_{t}\right)=O_{p}(1)$ and $n^{-1 / 2} \sum_{t=1}^{n} \varepsilon_{t} \delta\left(X_{t}\right)=O_{p}(1)$, by Hjajek's projection, the CauchySchwarz inequality, Chebyshev's inequality, and $E\left[\delta^{4}\left(X_{t}\right)\right] \leq C$.

For the $\hat{M}_{23}$ term in (A.44), using a second order Taylor series expansion, we have

$$
\begin{aligned}
\hat{M}_{23}= & \sum_{t=1}^{n} \varepsilon_{t} \sum_{s=1}^{n} w_{t s} \nabla_{\theta} g\left(X_{s}, \theta_{0}\right)\left(\hat{\theta}_{0}-\theta_{0}\right)+\frac{1}{2}\left(\hat{\theta}_{0}-\theta_{0}\right)^{\prime} \sum_{t=1}^{n} \varepsilon_{t} \sum_{s=1}^{n} w_{t s} \nabla_{\theta}^{2} g\left(X_{s}, \bar{\theta}\right)\left(\hat{\theta}_{0}-\theta_{0}\right) \\
& -\sum_{t=1}^{n} \tilde{m}_{h}\left(X_{t}\right) \sum_{s=1}^{n} w_{t s} \nabla_{\theta} g\left(X_{s}, \theta_{0}\right)\left(\hat{\theta}_{0}-\theta_{0}\right)-\frac{1}{2}\left(\hat{\theta}_{0}-\theta_{0}\right)^{\prime} \sum_{t=1}^{n} \tilde{m}_{h}\left(X_{t}\right) \sum_{s=1}^{n} \nabla_{\theta}^{2} g\left(X_{s}, \bar{\theta}\right)\left(\hat{\theta}_{0}-\theta_{0}\right) \\
= & O_{p}(1)+O_{p}(1)+O_{p}(1)+O_{p}(1)=O_{p}(1),
\end{aligned}
$$

given Assumptions A.1-A.3.

Next, for the $\hat{M}_{24}$ term in (A.44), we have

$$
\hat{M}_{24}=a_{n} \sum_{t=1}^{n} \varepsilon_{t} \sum_{s=1}^{n} w_{t s} \delta\left(X_{s}\right)-a_{n} \sum_{t=1}^{n} \tilde{m}_{h}\left(X_{t}\right) \sum_{s=1}^{n} w_{t s} \delta\left(X_{s}\right)
$$




$$
=O_{p}\left(n^{1 / 2} a_{n}\right)+O_{p}\left(n^{1 / 2} a_{n}\right)=o_{p}\left(h^{-p / 2}\right)
$$

by Hájek's projection, the Cauchy-Schwarz inequality, and $a_{n}=n^{-1 / 2} h^{-p / 4}$. Combining (A.36)-(A.48) together, we then obtain $S S R_{0}-S S R_{1}=\widetilde{S S R}_{0}-\widetilde{S S R}_{1}+o_{p}\left(h^{-p / 2}\right)$ under $\mathbb{H}_{n}\left(a_{n}\right)$.

Proof of Proposition 3.4: Proposition 3.4 follows from Lemmas 3.7 and 3.8 below.

Lemma 3.7: Put $\hat{H}_{\lambda}=n^{-1} \sum_{t=2}^{n} \sum_{s=1}^{t-1} H_{n}\left(Z_{t}, Z_{s}\right)$, where $Z_{t}=\left(\varepsilon_{t}, X_{t}^{\prime}\right)^{\prime}, H_{n}\left(Z_{t}, Z_{s}\right)=\varepsilon_{t} \varepsilon_{s} W_{h}\left(X_{t}, X_{s}\right)$, and

$$
W_{h}\left(X_{t}, X_{s}\right)=\left[\frac{1}{f\left(X_{t}\right)}+\frac{1}{f\left(X_{s}\right)}\right] \mathbf{K}_{h}\left(X_{t}-X_{s}\right)-\int \frac{\mathbf{K}_{h}\left(X_{t}-x\right) \mathbf{K}_{h}\left(X_{s}-x\right)}{f(x)} d x
$$

Suppose Assumptions A.1 and A.4 hold, and $h \propto n^{-\omega}$ for $\omega \in(0,1 / 2 p)$. Then

$$
\widetilde{S S R}_{0}-\widetilde{S S R}_{1}=h^{-p}\left[2 \mathbf{K}(0)-\int \mathbf{K}^{2}(\mathbf{u}) d \mathbf{u}\right] \int \sigma^{2}(x) d x+\hat{H}_{\lambda}+o_{p}\left(h^{-p / 2}\right) .
$$

Lemma 3.8: Suppose Assumptions A.1 and A.4 hold, and $h \propto n^{-\omega}$ for $\omega \in(0,1 / 2 p)$. Define

$$
V_{\lambda}=2 \int\left[\mathbf{K}(\mathbf{u})-\frac{1}{2} \int \mathbf{K}(\mathbf{v}) \mathbf{K}(\mathbf{u}+\mathbf{v}) d \mathbf{v}\right]^{2} d u \int \sigma^{4}(x) d x .
$$

Then $V_{\lambda}^{-1 / 2} h^{p / 2} \hat{H}_{\lambda} \stackrel{d}{\longrightarrow} N(\xi, 1)$.

Proof of Lemma 3.7: Let $\hat{F}_{n}(x)$ be the empirical distribution function of $X_{t}$. We first decompose

$$
\begin{aligned}
\widetilde{S S R}_{0}-\widetilde{S S R}_{1} & =2 \sum_{t=1}^{n} \varepsilon_{t} \tilde{m}_{h}\left(X_{t}\right)-\sum_{t=1}^{n} \tilde{m}_{h}^{2}\left(X_{t}\right) \\
& =2 \sum_{t=1}^{n} \varepsilon_{t} \tilde{m}_{h}\left(X_{t}\right)-n \int \tilde{m}_{h}^{2}(x) f(x) d x+n \int \tilde{m}_{h}^{2}(x) d\left[\hat{F}_{n}(x)-F(x)\right] \\
& =2 \sum_{t=1}^{n} \varepsilon_{t} \tilde{m}_{h}\left(X_{t}\right)-n \int \tilde{m}_{h}^{2}(x) f(x) d x+O_{p}\left(h^{-p}\right) O_{p}\left(n^{-1 / 2}(\ln n)^{2}\right)
\end{aligned}
$$

where we have made use of the well-known fact that $\sup _{x \in \mathbb{G}}\left|\hat{F}_{n}(x)-F(x)\right|=O_{p}\left(n^{-1 / 2}(\ln n)^{2}\right)$ (see, e.g., Bentkus, Gotse and Tikhomirov (1997)) under Assumption A.1 and $\int \tilde{m}_{h}^{2}(x) f(x) d x=O_{p}\left(n^{-1} h^{-p}\right)$ by Markov's inequality.

We now consider the first term in (A.49). We have

$$
\begin{aligned}
2 \sum_{t=1}^{n} \varepsilon_{t} \tilde{m}_{h}\left(X_{t}\right) & =2 \sum_{t=1}^{n} \varepsilon_{t} \sum_{s=1}^{n} \tilde{w}_{t s} \varepsilon_{s}+2 \sum_{t=1}^{n} \varepsilon_{t} \sum_{s=1}^{n}\left(w_{t s}-\tilde{w}_{t s}\right) \varepsilon_{s} \\
& =2 \sum_{t=1}^{n} \sum_{s=1}^{n} \tilde{w}_{t s} \varepsilon_{t} \varepsilon_{s}+O_{p}\left(h^{-p}\right) O_{p}\left(n^{-1 / 2} h^{-p / 2} \ln n+h^{2}\right)
\end{aligned}
$$

given $\sup _{x \in \mathbb{G}}|\hat{f}(x)-f(x)|=O_{p}\left(n^{-1 / 2} h^{-p / 2} \ln n+h^{2}\right)$ and Assumptions A.1 and A.4.

For the second term in (A.48), we have

$n \int \tilde{m}_{h}^{2}(x) f(x) d x$

$$
\begin{aligned}
& =n \int \frac{\left[n^{-1} \sum_{s=1}^{n} \varepsilon_{s} \mathbf{K}_{h}\left(X_{t}-X_{s}\right)\right]^{2}}{f(x)} d x+\int\left(n^{-1} \sum_{s=1}^{n} \varepsilon_{s} \mathbf{K}_{h}\left(X_{t}-X_{s}\right)\right)^{2}\left[\frac{1}{\hat{f}^{2}(x)}-\frac{1}{f^{2}(x)}\right] f(x) d x \\
& =n^{-1} \sum_{t=1}^{n} \sum_{s=1}^{n} \varepsilon_{t} \varepsilon_{s} \int \frac{\mathbf{K}_{h}\left(X_{t}-x\right) \mathbf{K}_{h}\left(X_{s}-x\right)}{f(x)} d x+o_{p}\left(h^{-p / 2}\right)
\end{aligned}
$$

where we have made use of the fact that $\sup _{x \in \mathbb{G}}|\hat{f}(x)-f(x)|=O_{p}\left(n^{-1 / 2} h^{-p / 2} \ln n+h^{2}\right)$ given Assumption A.2, 
and $h \propto n^{-\omega}$ for $\omega \in(0,1 / 2 p)$. It follows from (A.50) and (A.51) that

$$
\begin{aligned}
& 2 \sum_{t=1}^{n} \varepsilon_{t} \tilde{m}_{h}\left(X_{t}\right)-n \int \tilde{m}_{h}^{2}(x) f(x) d x=n^{-1} \sum_{t=1}^{n} \varepsilon_{t}^{2}\left[\frac{2 \mathbf{K}_{h}(0)}{f\left(X_{t}\right)}-\int \frac{\mathbf{K}_{h}^{2}\left(X_{t}-x\right)}{f(x)} d x\right] \\
& +n^{-1} \sum_{1 \leq s<t \leq n} \varepsilon_{t} \varepsilon_{s}\left\{\left[\frac{1}{f\left(X_{t}\right)}+\frac{1}{f\left(X_{s}\right)}\right] K_{h}\left(X_{t}-X_{s}\right)-\int \frac{\mathbf{K}_{h}\left(X_{t}-x\right) \mathbf{K}_{h}\left(X_{s}-x\right)}{f(x)} d x\right\}+o_{p}\left(h^{-p / 2}\right) \\
= & \hat{C}_{\lambda}+\hat{H}_{\lambda}+o_{p}\left(h^{-p / 2}\right), \text { say. }
\end{aligned}
$$

By change of variables, the law of iterated expectations, and Assumption A.1, we can obtain

$$
\begin{gathered}
E\left(\hat{C}_{\lambda}\right)=2 K_{h}(0) \int \sigma^{2}(x) d x-\iint \sigma^{2}(x) \frac{\mathbf{K}_{h}^{2}(y-x)}{f(x)} f(y) d x d y \\
=h^{-p}\left[2 \mathbf{K}(0)-\int \mathbf{K}^{2}(u) d u\right] \int \sigma^{2}(x) d x\left[1+O\left(h^{2}\right)\right] .
\end{gathered}
$$

On the other hand, by Chebyshev's inequality, we have

$$
\hat{C}_{\lambda}=E\left(\hat{C}_{\lambda}\right)+O_{p}\left(n^{-1 / 2} h^{-p}\right)
$$

where we have made use of the fact that $E\left(\hat{C}_{\lambda}-E \hat{C}_{\lambda}\right)^{2}=O_{p}\left(n^{-1} h^{-2 p}\right)$ given Assumption A.1. Combining (A.49),(A.52)-(A.54) and $p<4$ then yields the desired result. This completes the proof of Lemma 3.7.

Proof of Lemma 3.8: The proof of Lemma 3.8 is very similar to the proof of Lemma 3.4. We omit it here for space, except that we note that the asymptotic variance of $h^{p / 2} \hat{H}_{\lambda}$ is given by

$$
\begin{aligned}
n^{-2} \sum_{1 \leq s<t \leq n} h^{p} E\left[H_{n}^{2}\left(Z_{t}, Z_{s}\right)\right]= & h^{p} n^{-2} \sum_{1 \leq s<t \leq n} E\left[\varepsilon_{t}^{2} \varepsilon_{s}^{2} W_{h}^{2}\left(X_{t}, X_{s}\right)\right] \\
\rightarrow & 2 \int \mathbf{K}^{2}(u) d u \int \sigma^{4}(x) d x+\frac{1}{2} \int\left[\int \mathbf{K}(u) \mathbf{K}(u+v) d v\right]^{2} d u \int \sigma^{4}(x) d x \\
& -2 \iint \mathbf{K}(u) \mathbf{K}(v) \mathbf{K}(u+v) d u d v \int \sigma^{4}(x) d x \\
= & 2 \int\left[\mathbf{K}(u)-\frac{1}{2} \int \mathbf{K}(v) \mathbf{K}(u+v) d v\right]^{2} d u \int \sigma^{4}(x) d x \equiv V_{\lambda},
\end{aligned}
$$

by change of variables and Assumption A.1.

Proof of Theorem 4: The Pitman asymptotic relative efficiency of the $q_{n}$ test over the $\lambda_{n}$ test is the limit of sample sizes for the two tests to have the same asymptotic power at the same significance level, and under the same local alternative (see Pitman 1979, Ch 7). Specifically, supposed $n_{1}$ and $n_{2}$ are the sample sizes for the $\lambda_{n}$ test and the $q_{n}$ test respectively. Then Pitman's asymptotic relative efficiency of $q_{n}$ to $\lambda_{n}$ is defined as

$$
\operatorname{ARE}\left(q_{n}: \lambda_{n}\right)=\lim _{n_{1}, n_{2} \rightarrow \infty} \frac{n_{1}}{n_{2}}
$$

under the condition that $\lambda_{n}$ and $q_{n}$ have the same asymptotic power when the local alternatives $n_{1}^{-\frac{1}{2}} h_{1}^{-\frac{p}{4}} \delta_{1}(x) \sim$ $n_{2}^{\frac{1}{2}} h_{2}^{-\frac{p}{4}} \delta_{2}(x)$ in the sense that

$$
\lim _{n_{1}, n_{2} \rightarrow \infty} \frac{n_{1}^{-\frac{1}{2}} h_{1}^{-\frac{p}{4}} \delta_{1}(x)}{n_{2}^{\frac{1}{2}} h_{2}^{-\frac{p}{4}} \delta_{2}(x)}=1 .
$$

Given $h_{i}=c n_{i}^{-\omega}, i=1,2$, we have

$$
n_{1}^{-2 \gamma} E\left[\delta_{1}^{2}\left(X_{t}\right)\right] \sim n_{2}^{-2 \gamma} E\left[\delta_{2}^{2}\left(X_{t}\right)\right]
$$


where $\gamma=\frac{2-\omega p}{4}$. It follows that

$$
\lim _{n_{1}, n_{2} \rightarrow \infty}\left(\frac{n_{1}}{n_{2}}\right)^{2 \gamma}=\frac{E\left[\delta_{2}^{2}\left(X_{t}\right)\right]}{E\left[\delta_{1}^{2}\left(X_{t}\right)\right]}
$$

On the other hand, from Theorem 3, we have

$$
\frac{\gamma(K) \lambda_{n}-\mu_{n}}{\sqrt{2 \mu_{n}}} \stackrel{d}{\rightarrow} N(\xi, 1)
$$

under $\mathbb{H}_{n_{1}}\left(a_{n_{1}}\right): g_{0}\left(X_{t}\right)=g\left(X_{t}, \theta_{0}\right)+n_{1}^{-\frac{1}{2}} h_{1}^{-\frac{1}{4}} \delta_{1}\left(X_{t}\right)$, where $\xi=E\left[\delta_{1}^{2}\left(X_{t}\right)\right] /\left[2 \sigma^{-2} \sqrt{2 d(K) \int \sigma^{4}(x) d x}\right]$. Also, from Theorem 3, we have

$$
\frac{q_{n}-\nu_{n}}{\sqrt{2 \nu_{n}}} \stackrel{d}{\rightarrow} N(\psi, 1)
$$

under $\mathbb{H}_{n_{2}}\left(a_{n_{2}}\right): g_{0}\left(X_{t}\right)=g\left(X_{t}, \theta_{0}\right)+n_{2}^{-\frac{1}{2}} h_{2}^{-\frac{1}{4}} \delta_{2}\left(X_{t}\right)$, where $\psi=E\left[\delta_{2}^{2}\left(X_{t}\right)\right] / \sigma^{-2} \sqrt{2 b(K) \int \sigma^{4}(x) d x}$. To have the same asymptotic power, the noncentrality parameters must be equal; namely $\xi=\psi$, or

$$
\frac{E\left[\delta_{1}^{2}\left(X_{t}\right)\right]}{2 \sqrt{2 d(K) \int \sigma^{4}(x) d x}}=\frac{E\left[\delta_{2}^{2}\left(X_{t}\right)\right]}{\sqrt{2 b(K) \int \sigma^{4}(x) d x}} .
$$

Combining (A.55) and (A.56) yields

$$
\begin{aligned}
A R E\left(q_{n}, \lambda_{n}\right) & \equiv \lim _{n_{1}, n_{2} \rightarrow \infty} \frac{n_{1}}{n_{2}}=\left[\frac{2 \sqrt{d(K)}}{\sqrt{b(K)}}\right]^{\frac{1}{2 \gamma}}=\left[\frac{4 d(K)}{b(K)}\right]^{\frac{1}{4 \gamma}} \\
& =\left[\frac{\int\left(2 \mathbf{K}(u)-\int \mathbf{K}(u) \mathbf{K}(u+v) d u\right)^{2} d v}{\int\left(\int \mathbf{K}(u) \mathbf{K}(u+v) d u\right)^{2} d v}\right]^{\frac{1}{2-\omega p}} .
\end{aligned}
$$

Finally, we shall show $\operatorname{ARE}\left(q_{n}: \lambda_{n}\right) \geq 1$ for any positive kernels with $K(\cdot) \leq 1$. For this purpose, it suffices to show

$$
\int\left[2 \mathbf{K}(u)-\int \mathbf{K}(u) \mathbf{K}(u+v) d u\right]^{2} d v \geq \int\left[\int \mathbf{K}(u) \mathbf{K}(u+v) d u\right]^{2} d v
$$

or equivalently,

$$
\int \mathbf{K}^{2}(v) d v \geq \iint \mathbf{K}(u) \mathbf{K}(v) \mathbf{K}(u+v) d u d v
$$

This last inequality follows from a lemma of Zhang and Dette (2004, Lemma 2). This completes the proof. 
B. Simulation Results for Tests with the Bandwidth $h=S_{X} n^{-1 / 5}$

TABLE S.1. Empirical Sizes of Tests Using Asymptotic Critical Values

\begin{tabular}{|c|c|c|c|c|c|c|c|c|c|c|c|c|c|c|c|c|c|c|}
\hline & \multicolumn{2}{|c|}{$q_{n}$} & \multicolumn{2}{|c|}{$q_{n}^{0}$} & \multicolumn{2}{|c|}{ GLR } & \multicolumn{2}{|c|}{$q_{n}$} & \multicolumn{2}{|c|}{$q_{n}^{0}$} & \multicolumn{2}{|c|}{$G L R$} & \multicolumn{2}{|c|}{$q_{n}$} & \multicolumn{2}{|c|}{$q_{n}^{0}$} & \multicolumn{2}{|c|}{$G L R$} \\
\hline$(a, \beta)$ & $10 \%$ & $5 \%$ & $10 \%$ & $5 \%$ & $10 \%$ & $5 \%$ & $10 \%$ & $5 \%$ & $10 \%$ & $5 \%$ & $10 \%$ & $5 \%$ & $10 \%$ & $5 \%$ & $10 \%$ & $5 \%$ & $10 \%$ & $5 \%$ \\
\hline \multicolumn{7}{|c|}{$n=100$} & \multicolumn{6}{|c|}{$n=250$} & \multicolumn{6}{|c|}{$n=500$} \\
\hline & \multicolumn{18}{|c|}{ DGP S.1: i.i.d. Normal Errors } \\
\hline$(0.0,1.0)$ & 7.4 & 4.4 & 4.5 & 2.5 & 7.2 & 4.0 & 4.7 & 3.3 & 4.0 & 2.4 & 7.7 & 4.1 & 5.7 & 3.8 & 5.1 & 3.3 & 7.3 & 3.6 \\
\hline$(0.2,1.0)$ & 7.6 & 4.3 & 4.4 & 2.7 & 7.2 & 4.0 & 4.7 & 3.4 & 4.0 & 2.2 & 7.7 & 4.1 & 5.7 & 3.8 & 5.0 & 3.5 & 7.3 & 3.6 \\
\hline$(0.5,1.0)$ & 7.5 & 4.7 & 4.8 & 3.1 & 7.2 & 4.0 & 4.9 & 3.3 & 3.8 & 2.2 & 7.7 & 4.1 & 6.0 & 4.1 & 5.1 & 3.5 & 7.3 & 3.6 \\
\hline \multirow[t]{2}{*}{$(1.0,1.0)$} & 7.8 & 5.4 & 5.6 & 4.2 & 7.2 & 4.0 & 5.1 & 3.3 & 3.8 & 2.7 & 7.7 & 4.1 & 6.1 & 4.1 & 5.3 & 3.8 & 7.3 & 3.6 \\
\hline & \multicolumn{18}{|c|}{ DGP S.2: i.i.d. Student- $t_{5}$ Errors } \\
\hline$(0.0,1.0)$ & 5.8 & 3.7 & 3.6 & 2.2 & 6.2 & 3.4 & 5.1 & 2.2 & 3.4 & 1.7 & 6.3 & 3.0 & 4.2 & 2.6 & 4.1 & 2.3 & 5.7 & 3.0 \\
\hline$(0.2,1.0)$ & 5.8 & 3.7 & 3.6 & 2.2 & 6.2 & 3.4 & 5.2 & 2.6 & 3.3 & 2.0 & 6.3 & 3.0 & 4.3 & 2.7 & 4.0 & 2.4 & 5.7 & 3.0 \\
\hline$(0.5,1.0)$ & 5.8 & 3.9 & 4.0 & 2.3 & 6.2 & 3.4 & 5.1 & 2.8 & 3.4 & 2.1 & 6.3 & 3.0 & 4.6 & 2.6 & 4.1 & 2.5 & 5.7 & 3.0 \\
\hline \multirow[t]{2}{*}{$(1.0,1.0)$} & 6.8 & 4.7 & 4.7 & 2.7 & 6.2 & 3.4 & 5.3 & 3.1 & 3.8 & 2.7 & 6.3 & 3.0 & 4.6 & 2.5 & 4.5 & 2.5 & 5.7 & 3.0 \\
\hline & \multicolumn{18}{|c|}{ DGP S.3: i.i.d. Uniform Errors } \\
\hline$(0.0,1.0)$ & 5.8 & 4.4 & 4.3 & 1.9 & 5.2 & 3.2 & 5.9 & 3.6 & 4.4 & 2.7 & 6.3 & 3.2 & 5.7 & 3.6 & 5.1 & 3.3 & 5.9 & 2.6 \\
\hline$(0.2,1.0)$ & 5.7 & 4.6 & 4.3 & 1.8 & 5.2 & 3.2 & 6.2 & 3.4 & 4.4 & 2.7 & 6.3 & 3.2 & 5.7 & 3.9 & 5.3 & 3.1 & 5.9 & 2.6 \\
\hline$(0.5,1.0)$ & 6.3 & 4.5 & 4.1 & 2.0 & 5.2 & 3.2 & 5.9 & 3.8 & 4.7 & 2.7 & 6.3 & 3.2 & 5.8 & 3.8 & 5.4 & 3.3 & 5.9 & 2.6 \\
\hline \multirow[t]{2}{*}{$(1.0,1.0)$} & 8.0 & 5.5 & 5.4 & 2.7 & 5.2 & 3.2 & 6.1 & 4.4 & 4.7 & 3.1 & 6.3 & 3.2 & 6.5 & 4.0 & 5.5 & 3.4 & 5.9 & 2.6 \\
\hline & \multicolumn{18}{|c|}{ DGP S.4: i.i.d. Log-Normal Errors } \\
\hline$(0.0,1.0)$ & 8.0 & 6.3 & 5.9 & 3.8 & 7.5 & 5.8 & 4.8 & 3.0 & 3.9 & 2.3 & 6.2 & 3.5 & 6.2 & 4.2 & 5.6 & 3.4 & 6.5 & 4.2 \\
\hline$(0.2,1.0)$ & 8.5 & 6.8 & 6.5 & 4.6 & 7.5 & 5.8 & 5.0 & 3.3 & 4.2 & 2.4 & 6.2 & 3.5 & 6.2 & 4.8 & 5.7 & 3.7 & 6.5 & 4.2 \\
\hline$(0.5,1.0)$ & 9.3 & 7.5 & 7.2 & 5.3 & 7.5 & 5.8 & 5.9 & 4.0 & 4.8 & 3.2 & 6.2 & 3.5 & 6.7 & 5.3 & 6.2 & 4.3 & 6.5 & 4.2 \\
\hline$(1.0,1.0)$ & 10.9 & 8.9 & 9.1 & 7.0 & 7.5 & 5.8 & 7.6 & 5.2 & 6.0 & 4.3 & 6.2 & 3.5 & 7.7 & 6.0 & 6.8 & 5.3 & 6.5 & 4.2 \\
\hline \multicolumn{19}{|c|}{ DGP S.5:i.i.d.Chi-Square Errors } \\
\hline$(0.0,1.0)$ & 7.3 & 5.0 & 4.7 & 3.5 & 6.7 & 4.6 & 5.4 & 3.3 & 4.0 & 1.9 & 5.8 & 3.7 & 4.5 & 2.7 & 3.9 & 2.4 & 4.8 & 2.8 \\
\hline$(0.2,1.0)$ & 7.5 & 5.3 & 5.1 & 3.7 & 6.7 & 4.6 & 5.7 & 3.4 & 4.2 & 2.7 & 5.8 & 3.7 & 4.6 & 2.8 & 4.1 & 2.5 & 4.8 & 2.8 \\
\hline$(0.5,1.0)$ & 8.1 & 6.2 & 5.9 & 4.1 & 6.7 & 4.6 & 6.1 & 3.9 & 5.0 & 3.0 & 5.8 & 3.7 & 4.7 & 3.2 & 4.3 & 2.6 & 4.8 & 2.8 \\
\hline$(1.0,1.0)$ & 9.1 & 7.3 & 7.6 & 5.8 & 6.7 & 4.6 & 6.5 & 4.6 & 5.8 & 3.7 & 5.8 & 3.7 & 5.8 & 3.9 & 4.9 & 3.3 & 4.8 & 2.8 \\
\hline
\end{tabular}

Notes : (i) 1000 iterations;

(ii) $G L R$, the generalized likelihood ratio test, $q_{n}$ and $q_{n}^{0}$, loss function-based tests;

(iii) $q_{n}$ is standardized by $S S R_{1}$, the sum of squared residuals of the nonparametric regression estimates, and $q_{n}^{0}$ is standardized by $S S R_{0}$, the sum of squared residuals of the null linear model;

(iv) The uniform kernel is used for $G L R, q_{n}$ and $q_{n}^{0}$; the bandwidth $h=S_{X} n^{-1 / 5}$, where $S_{X}$ is the sample standard deviation of $\left\{X_{t}\right\}_{t=1}^{n}$;

(v) The $q_{n}$ tests are based on the linex loss function: $d(z)=\frac{\beta}{\alpha^{2}}[\exp (\alpha z)-1-\alpha z]$;

(vi) $Y_{t}=1+X_{t}+\varepsilon_{t}, X_{t}=0.5 X_{t-1}+v_{t}, v_{t} \sim i . i . d . N(0,1)$, where DGP S.1: $\varepsilon_{i} \sim$ i.i.d. $N(0,1)$; DGP S.2: $\varepsilon_{i}$ $\sim$ i.i.d. Student- $t_{5}$; DGP S.3: $\varepsilon_{i} \sim$ i.i.d. $U[0,1]$; DGP S.4: $\varepsilon_{i} \sim$ i.i.d. $\log N(0,1)$; DGP S.5: $\varepsilon_{i} \sim i . i . d . \chi_{1}^{2}$. 
TABLE S.2. Empirical Sizes of Tests Using Bootstrap Critical Values

\begin{tabular}{|c|c|c|c|c|c|c|c|c|c|c|c|c|c|c|c|c|c|c|}
\hline \multirow[b]{2}{*}{$(a, \beta)$} & \multicolumn{2}{|c|}{$q_{n}$} & \multicolumn{2}{|c|}{$q_{n}^{0}$} & \multicolumn{2}{|c|}{$G L R$} & \multicolumn{2}{|c|}{$q_{n}$} & \multicolumn{2}{|c|}{$q_{n}^{0}$} & \multicolumn{2}{|c|}{$G L R$} & \multicolumn{2}{|c|}{$q_{n}$} & \multicolumn{2}{|c|}{$q_{n}^{0}$} & \multicolumn{2}{|c|}{$G L R$} \\
\hline & $10 \%$ & $5 \%$ & $10 \%$ & $5 \%$ & $10 \%$ & $5 \%$ & $10 \%$ & $5 \%$ & $10 \%$ & $5 \%$ & $10 \%$ & $5 \%$ & $10 \%$ & $5 \%$ & $10 \%$ & $5 \%$ & $10 \%$ & $5 \%$ \\
\hline \multicolumn{7}{|c|}{$n=100$} & \multicolumn{6}{|c|}{$n=250$} & \multicolumn{6}{|c|}{$n=500$} \\
\hline & \multicolumn{18}{|c|}{ DGP S.1: i.i.d. Normal Errors } \\
\hline$(0.0,1.0)$ & 10.1 & 5.2 & 10.2 & 4.9 & 10.6 & 4.9 & 11.8 & 5.8 & 11.6 & 5.8 & 11.7 & 5.3 & 12.0 & 5.8 & 12.5 & 5.6 & 11.2 & 6.7 \\
\hline$(0.2,1.0)$ & 10.0 & 5.4 & 10.1 & 5.3 & 10.6 & 4.9 & 11.9 & 5.6 & 11.6 & 5.8 & 11.7 & 5.3 & 12.2 & 5.4 & 12.0 & 5.4 & 11.2 & 6.7 \\
\hline$(0.5,1.0)$ & 9.5 & 5.2 & 9.2 & 5.3 & 10.6 & 4.9 & 11.6 & 6.0 & 11.4 & 5.8 & 11.7 & 5.3 & 11.8 & 5.5 & 11.9 & 5.6 & 11.2 & 6.7 \\
\hline \multirow[t]{2}{*}{$(1.0,1.0)$} & 8.9 & 5.0 & 9.4 & 5.0 & 10.6 & 4.9 & 10.9 & 5.6 & 10.9 & 5.4 & 11.7 & 5.3 & 12.4 & 5.7 & 12.3 & 5.5 & 11.2 & 6.7 \\
\hline & \multicolumn{18}{|c|}{ DGP S.2: i.i.d. Student- $t_{5}$ Errors } \\
\hline$(0.0,1.0)$ & 8.4 & 4.0 & 8.2 & 3.9 & 9.3 & 4.5 & 8.8 & 4.2 & 8.8 & 3.9 & 10.8 & 5.0 & 10.3 & 5.4 & 10.4 & 4.9 & 9.5 & 4.9 \\
\hline$(0.2,1.0)$ & 8.7 & 3.9 & 8.6 & 4.0 & 9.3 & 4.5 & 8.7 & 4.1 & 8.8 & 4.3 & 10.8 & 5.0 & 10.3 & 5.6 & 10.2 & 5.6 & 9.5 & 4.9 \\
\hline$(0.5,1.0)$ & 8.6 & 4.5 & 8.7 & 4.2 & 9.3 & 4.5 & 8.5 & 4.4 & 8.6 & 4.4 & 10.8 & 5.0 & 10.1 & 5.6 & 10.3 & 5.6 & 9.5 & 4.9 \\
\hline \multirow[t]{2}{*}{$(1.0,1.0)$} & 9.0 & 4.5 & 9.3 & 4.2 & 9.3 & 4.5 & 8.3 & 4.2 & 8.1 & 4.0 & 10.8 & 5.0 & 9.9 & 5.2 & 10.1 & 5.3 & 9.5 & 4.9 \\
\hline & \multicolumn{18}{|c|}{ DGP S.3: i.i.d. Uniform Errors } \\
\hline$(0.0,1.0)$ & 9.7 & 5.4 & 9.6 & 5.6 & 10.1 & 4.9 & 11.8 & 6.0 & 11.7 & 6.1 & 11.3 & 5.8 & 9.7 & 6.4 & 9.6 & 6.2 & 10.5 & 5.2 \\
\hline$(0.2,1.0)$ & 9.4 & 5.6 & 9.3 & 5.9 & 10.1 & 4.9 & 12.0 & 5.9 & 11.9 & 5.9 & 11.3 & 5.8 & 9.7 & 6.4 & 9.8 & 6.1 & 10.5 & 5.2 \\
\hline$(0.5,1.0)$ & 9.2 & 5.6 & 9.3 & 6.1 & 10.1 & 4.9 & 11.4 & 5.8 & 11.4 & 6.1 & 11.3 & 5.8 & 9.8 & 6.5 & 10.1 & 6.1 & 10.5 & 5.2 \\
\hline \multirow[t]{2}{*}{$(1.0,1.0)$} & 10.1 & 5.7 & 9.9 & 5.6 & 10.1 & 4.9 & 11.9 & 5.9 & 11.7 & 6.1 & 11.3 & 5.8 & 10.3 & 6.6 & 10.2 & 6.6 & 10.5 & 5.2 \\
\hline & \multicolumn{18}{|c|}{ DGP S.4: i.i.d. Log-Normal Errors } \\
\hline$(0.0,1.0)$ & 10.4 & 5.7 & 10.2 & 4.9 & 10.7 & 6.6 & 10.3 & 4.5 & 10.4 & 4.2 & 10.9 & 5.3 & 9.8 & 5.3 & 10.2 & 5.2 & 11.4 & 5.1 \\
\hline$(0.2,1.0)$ & 10.8 & 5.8 & 9.8 & 5.0 & 10.7 & 6.6 & 10.5 & 4.4 & 10.8 & 4.2 & 10.9 & 5.3 & 10.0 & 5.4 & 10.2 & 5.3 & 11.4 & 5.1 \\
\hline$(0.5,1.0)$ & 11.1 & 5.9 & 9.9 & 5.3 & 10.7 & 6.6 & 10.6 & 4.3 & 10.5 & 4.2 & 10.9 & 5.3 & 10.1 & 5.2 & 10.2 & 5.3 & 11.4 & 5.1 \\
\hline$(1.0,1.0)$ & 10.7 & 6.2 & 10.2 & 5.9 & 10.7 & 6.6 & 10.5 & 4.4 & 10.5 & 4.2 & 10.9 & 5.3 & 10.3 & 5.4 & 10.6 & 5.5 & 11.4 & 5.1 \\
\hline \multicolumn{19}{|c|}{ DGP S.5: i.i.d. Chi-Square Errors } \\
\hline$(0.0,1.0)$ & 8.9 & 4.1 & 8.8 & 4.0 & 10.5 & 5.2 & 10.2 & 4.8 & 10.0 & 4.8 & 9.8 & 5.1 & 8.0 & 3.8 & 8.5 & 3.7 & 8.0 & 4.2 \\
\hline$(0.2,1.0)$ & 8.6 & 4.8 & 8.7 & 4.1 & 10.5 & 5.2 & 9.9 & 4.7 & 9.6 & 4.7 & 9.8 & 5.1 & 8.5 & 4.1 & 8.5 & 4.1 & 8.0 & 4.2 \\
\hline$(0.5,1.0)$ & 9.0 & 4.3 & 8.6 & 4.1 & 10.5 & 5.2 & 10.0 & 4.4 & 9.7 & 4.3 & 9.8 & 5.1 & 8.0 & 3.9 & 8.2 & 4.0 & 8.0 & 4.2 \\
\hline$(1.0,1.0)$ & 8.8 & 4.5 & 8.5 & 4.3 & 10.5 & 5.2 & 9.7 & 4.7 & 9.9 & 4.2 & 9.8 & 5.1 & 8.6 & 3.8 & 8.7 & 3.7 & 8.0 & 4.2 \\
\hline
\end{tabular}

Notes : (i) 1000 iterations;

(ii) $G L R$, the generalized likelihood ratio test, $q_{n}$ and $q_{n}^{0}$, loss function-based tests;

(iii) $q_{n}$ is standardized by $S S R_{1}$, the sum of squared residuals of the nonparametric regression estimates, and $q_{n}^{0}$ is standardized by $S S R_{0}$, the sum of squared residuals of the null linear model;

(iv) The uniform kernel is used for $G L R, q_{n}$ and $q_{n}^{0}$; the bandwidth $h=S_{X} n^{-1 / 5}$, where $S_{X}$ is the sample standard deviation of $\left\{X_{t}\right\}_{t=1}^{n}$;

(v) The $q_{n}$ tests are based on the linex loss function: $d(z)=\frac{\beta}{\alpha^{2}}[\exp (\alpha z)-1-\alpha z]$;

(vi) $Y_{t}=1+X_{t}+\varepsilon_{t}, X_{t}=0.5 X_{t-1}+v_{t}, v_{t} \sim$ i.i.d.N(0,1), where DGP S.1: $\varepsilon_{i} \sim$ i.i.d. $N(0,1)$; DGP S.2: $\varepsilon_{i}$ $\sim$ i.i.d. Student- $t_{5}$; DGP S.3: $\varepsilon_{i} \sim$ i.i.d. $U[0,1]$; DGP S.4: $\varepsilon_{i} \sim$ i.i.d. $\log N(0,1)$; DGP S.5: $\varepsilon_{i} \sim$ i.i.d. $\chi_{1}^{2}$. 
TABLE S.3. Empirical Powers of Tests Using Bootstrap Critical Values

\begin{tabular}{|c|c|c|c|c|c|c|c|c|c|c|c|c|c|c|c|c|c|c|c|}
\hline & & \multicolumn{18}{|c|}{ DGP P.1: Quadratic Regression } \\
\hline & & \multicolumn{2}{|c|}{$q_{n}$} & \multicolumn{2}{|c|}{$q_{n}^{0}$} & \multicolumn{2}{|c|}{ GLR } & \multicolumn{2}{|c|}{$q_{n}$} & \multicolumn{2}{|c|}{$q_{n}^{0}$} & \multicolumn{2}{|c|}{ GLR } & \multicolumn{2}{|c|}{$q_{n}$} & \multicolumn{2}{|c|}{ 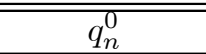 } & \multicolumn{2}{|c|}{$\overline{G L L R}$} \\
\hline \multirow[t]{2}{*}{$(a, \beta)$} & $\theta$ & $10 \%$ & $5 \%$ & $10 \%$ & $5 \%$ & $10 \%$ & $5 \%$ & $10 \%$ & $5 \%$ & $10 \%$ & $5 \%$ & $10 \%$ & $5 \%$ & $10 \%$ & $5 \%$ & $10 \%$ & $5 \%$ & $10 \%$ & $5 \%$ \\
\hline & & \multicolumn{6}{|c|}{$n=100$} & \multicolumn{6}{|c|}{$n=250$} & \multicolumn{6}{|c|}{$n=500$} \\
\hline \multirow[t]{5}{*}{$(0.0,1.0)$} & 0.1 & 20.7 & 13.2 & 20.5 & 13.4 & 19.2 & 11.7 & 38.9 & 27.3 & 39.1 & 27.2 & 31.1 & 22.2 & 59.1 & 48.4 & 65.4 & 54.6 & 52.4 & 38.8 \\
\hline & 0.2 & 53.6 & 39.4 & 53.3 & 39.8 & 44.3 & 32.3 & 89.5 & 83.6 & 89.7 & 84.4 & 80.6 & 70.4 & 99.2 & 98.7 & 99.4 & 99.1 & 98.0 & 97.1 \\
\hline & 0.3 & 83.3 & 75.5 & 83.3 & 75.4 & 76.4 & 66.0 & 99.7 & 99.1 & 99.7 & 99.1 & 98.5 & 96.4 & 99.3 & 98.6 & 100.0 & 99.8 & 100.0 & 100.0 \\
\hline & 0.5 & 99.0 & 98.2 & 99.0 & 98.2 & 98.1 & 97.0 & 100.0 & 100.0 & 100.0 & 100.0 & 100.0 & 100.0 & 100.0 & 100.0 & 100.0 & 100.0 & 100.0 & 100.0 \\
\hline & 1.0 & 100.0 .0 & 100.0 & 100.0 & 100.0 & 100.0 & 100.0 & 100.0 & 100.0 & 100.0 & 100.0 & 100.0 & 100.0 & 100.0 & 100.0 & 100.0 & 100.0 & 100.0 & 100.0 \\
\hline \multirow[t]{5}{*}{$(0.2,1.0)$} & 0.1 & 21.3 & 13.9 & 21.0 & 14.3 & 19.2 & 11.7 & 40.0 & 28.2 & 40.0 & 28.0 & 31.1 & 22.2 & 66.3 & 55.4 & 66.5 & 55.5 & 52.4 & 38.8 \\
\hline & 0.2 & 55.2 & 40.9 & 55.6 & 41.8 & 44.3 & 32.3 & 89.9 & 84.7 & 90.2 & 85.5 & 80.6 & 70.4 & 99.5 & 99.1 & 99.5 & 99.2 & 98.0 & 97.1 \\
\hline & 0.3 & 84.5 & 76.9 & 84.8 & 76.9 & 76.4 & 66.0 & 99.8 & 99.3 & 99.8 & 99.3 & 98.5 & 96.4 & 100.0 & 100.0 & 100.0 & 100.0 & 100.0 & 100.0 \\
\hline & 0.5 & 99.5 & 98.5 & 99.1 & 98.4 & 98.1 & 97.0 & 100.0 & 100.0 & 100.0 & 100.0 & 100.0 & 100.0 & 100.0 & 100.0 & 100.0 & 100.0 & 100.0 & 100.0 \\
\hline & 1.0 & 100.0 & 100.0 & 100.0 & 100.0 & 100.0 & 100.0 & 100.0 & 100.0 & 100.0 & 100.0 & 100.0 & 100.0 & 100.0 & 100.0 & 100.0 & 100.0 & 100.0 & 100.0 \\
\hline \multirow[t]{5}{*}{$(0.5,1.0)$} & 0.1 & 22.7 & 14.8 & 22.7 & 15.2 & 19.2 & 11.7 & 41.6 & 29.2 & 41.8 & 29.5 & 31.1 & 22.2 & 67.1 & 56.8 & 67.3 & 57.0 & 52.4 & 38.8 \\
\hline & 0.2 & 57.4 & 43.3 & 57.2 & 44.4 & 44.3 & 32.3 & 90.7 & 86.6 & 91.0 & 87.0 & 80.6 & 70.4 & 99.7 & 99.2 & 99.7 & 99.2 & 98.0 & 97.1 \\
\hline & 0.3 & 85.8 & 78.3 & 85.9 & 78.2 & 76.4 & 66.0 & 99.8 & 99.5 & 99.8 & 99.5 & 98.5 & 96.4 & 100.0 & 100.0 & 100.0 & 100.0 & 100.0 & 100.0 \\
\hline & 0.5 & 99.5 & 98.9 & 99.5 & 98.8 & 98.1 & 97.0 & 100.0 & 100.0 & 100.0 & 100.0 & 100.0 & 100.0 & 100.0 & 100.0 & 100.0 & 100.0 & 100.0 & 100.0 \\
\hline & 1.0 & 100.0 & 100.0 & 100.0 & 100.0 & 100.0 & 100.0 & 100.0 & 100.0 & 100.0 & 100.0 & 100.0 & 100.0 & 100.0 & 100.0 & 100.0 & 100.0 & 100.0 & 100.0 \\
\hline \multirow[t]{5}{*}{$(1.0,1.0)$} & 0.1 & 25.0 & 15.9 & 25.7 & 16.3 & 19.2 & 11.7 & 44.2 & 31.7 & 44.4 & 32.1 & 31.1 & 22.2 & 69.2 & 59.0 & 69.4 & 58.8 & 52.4 & 38.8 \\
\hline & 0.2 & 59.5 & 47.3 & 59.7 & 48.2 & 44.3 & 32.3 & 91.8 & 87.4 & 91.7 & 87.6 & 80.6 & 70.4 & 99.7 & 99.3 & 99.7 & 99.3 & 98.0 & 97.1 \\
\hline & 0.3 & 86.7 & 79.8 & 86.8 & 80.3 & 76.4 & 66.0 & 99.8 & 99.6 & 99.9 & 99.7 & 98.5 & 96.4 & 100.0 & 100.0 & 100.0 & 100.0 & 100.0 & 100.0 \\
\hline & 0.5 & 99.6 & 98.9 & 99.5 & 98.7 & 98.1 & 97.0 & 100.0 & 100.0 & 100.0 & 100.0 & 100.0 & 100.0 & 100.0 & 100.0 & 100.0 & 100.0 & 100.0 & 100.0 \\
\hline & 1.0 & 100.0 & 100.0 & 100.0 & 100.0 & 100.0 & 100.0 & 100.0 & 100.0 & 100.0 & 100.0 & 100.0 & 100.0 & 100.0 & 100.0 & 100.0 & 100.0 & 100.0 & 100.0 \\
\hline
\end{tabular}

Notes : (i) 1000 iterations;

(ii) $G L R$, the generalized likelihood ratio test, $q_{n}$ and $q_{n}^{0}$, loss function-based tests;

(iii) $q_{n}$ is standardized by $S S R_{1}$, the sum of squared residuals of the nonparametric regression estimates, and $q_{n}^{0}$ is standardized by $S S R_{0}$, the sum of squared residuals of the null linear model;

(iv) The uniform kernel is used for $G L R, q_{n}$ and $q_{n}^{0}$; the bandwidth $h=S_{X} n^{-1 / 5}$, where $S_{X}$ is the sample standard deviation of $\left\{X_{t}\right\}_{t=1}^{n}$;

(v) The $q_{n}$ tests are based on the linex loss function: $d(z)=\frac{\beta}{\alpha^{2}}[\exp (\alpha z)-1-\alpha z]$;

(vi) DGP P.1, $Y_{t}=1+X_{t}+\theta X_{t}^{2}+\varepsilon_{t}$, where $\left\{\varepsilon_{i}\right\} \sim$ i.i.d.N $(0,1)$. 
TABLE S.4. Empirical Powers of Tests Using Bootstrap Critical Values

\begin{tabular}{|c|c|c|c|c|c|c|c|c|c|c|c|c|c|c|c|c|c|c|c|}
\hline & & \multicolumn{18}{|c|}{ DGP P.2: Threshold Regression } \\
\hline & & \multicolumn{2}{|c|}{$q_{n}$} & \multicolumn{2}{|c|}{$q_{n}^{0}$} & \multicolumn{2}{|c|}{$G L R$} & \multicolumn{2}{|c|}{$q_{n}$} & \multicolumn{2}{|c|}{$q_{n}^{0}$} & \multicolumn{2}{|c|}{ GLR } & \multicolumn{2}{|c|}{$q_{n}$} & \multicolumn{2}{|c|}{$q_{n}^{0}$} & \multicolumn{2}{|c|}{$\overline{G L L R}$} \\
\hline \multirow[t]{2}{*}{$(a, \beta)$} & $\theta$ & $10 \%$ & $5 \%$ & $10 \%$ & $5 \%$ & $10 \%$ & $5 \%$ & $10 \%$ & $5 \%$ & $10 \%$ & $5 \%$ & $10 \%$ & $5 \%$ & $10 \%$ & $5 \%$ & $10 \%$ & $5 \%$ & $10 \%$ & $5 \%$ \\
\hline & & \multicolumn{6}{|c|}{$n=100$} & \multicolumn{6}{|c|}{$n=250$} & \multicolumn{6}{|c|}{$n=500$} \\
\hline \multirow[t]{6}{*}{$(0.0,1.0)$} & -1.0 & 72.7 & 60.4 & 73.2 & 61.3 & 61.8 & 48.5 & 97.7 & 95.6 & 97.5 & 95. & 94.3 & 91.0 & 100.0 & 100.0 & 100.0 & 100.0 & 100.0 & 100.0 \\
\hline & -0.5 & 26.9 & 17.4 & 27.0 & 17.8 & 24.0 & 14.4 & 52.5 & 40.3 & 53.0 & 40. & 42.5 & 31.9 & 84.6 & 73.2 & 84.6 & 73.3 & 68.9 & 57.5 \\
\hline & -0.2 & 12.3 & 7.8 & 12.5 & 7.9 & 12.2 & 5.9 & 17.9 & 10.7 & 17.3 & 10. & 15.4 & 9.2 & 25.4 & 15.7 & 25.4 & 15.6 & 21.1 & 11.8 \\
\hline & 0.2 & 12.7 & 6.6 & 12.5 & 6.6 & 11.7 & 6.6 & 16.8 & 10.2 & 17.2 & 10. & 15.9 & 8.5 & 24.6 & 15.1 & 24.7 & 15.1 & 20.1 & 12.4 \\
\hline & 0.5 & 27.1 & 17.9 & 27.6 & 18.1 & 22.1 & 13.6 & 53.7 & 41.6 & 54.1 & 41. & 41.3 & 30.6 & 80.5 & 70.4 & 80.5 & 70.0 & 66.0 & 54.3 \\
\hline & 1.0 & 71.8 & 59.2 & 71.7 & 59.9 & 58.6 & 45.8 & 97.9 & 95.9 & 98.0 & 95. & 92.7 & 88.8 & 100.0 & 100.0 & 100.0 & 100.0 & 99.9 & 99.6 \\
\hline \multirow[t]{6}{*}{$(0.2,1.0)$} & -1.0 & 74.3 & 61.4 & 74.3 & 62.5 & 61.8 & 48.5 & 98.0 & 96.0 & 97.9 & 96. & 94.3 & 91.0 & 100.0 & 100.0 & 100.0 & 100.0 & 100.0 & 100.0 \\
\hline & -0.5 & 27.8 & 18.3 & 28.4 & 18.5 & 24.0 & 14.4 & 53.7 & 41.7 & 54.2 & 41. & 42.5 & 31.9 & 84.9 & 73.9 & 84.9 & 74.1 & 68.9 & 57.5 \\
\hline & -0.2 & 12.6 & 8.2 & 13.2 & 8.4 & 12.2 & 5.9 & 18.1 & 10.9 & 18.3 & 11. & 15.4 & 9.2 & 25.8 & 15.9 & 25.6 & 15.9 & 21.1 & 11.8 \\
\hline & 0.2 & 11.8 & 6.6 & 12.6 & 6.5 & 11.7 & 6.6 & 16.5 & 10.0 & 16.6 & 9.9 & 15.9 & 8.5 & 24.4 & 14.6 & 24.5 & 14.9 & 20.1 & 12.4 \\
\hline & 0.5 & 26.2 & 16.9 & 26.3 & 16.5 & 22.1 & 13.6 & 53.1 & 40.5 & 53.0 & 40. & 41.3 & 30.6 & 79.8 & 69.4 & 80.0 & 69.7 & 66.0 & 54.3 \\
\hline & 1.0 & 70.6 & 58.6 & 70.2 & 58.5 & 58.6 & 45.8 & 97.8 & 95.7 & 97.6 & 95. & 92.7 & 88.8 & 100.0 & 99.9 & 100.0 & 99.9 & 99.9 & 99.6 \\
\hline \multirow[t]{6}{*}{$(0.5,1.0)$} & -1.0 & 75.1 & 63.1 & 75.3 & 64.2 & 61.8 & 48.5 & 98.1 & 95.9 & 97.9 & 96. & 94.3 & 91.0 & 100.0 & 100.0 & 100.0 & 100.0 & 100.0 & 100.0 \\
\hline & -0.5 & 29.4 & 19.8 & 29.7 & 19.4 & 24.0 & 14.4 & 55.0 & 42.7 & 55.8 & 42. & 42.5 & 31.9 & 85.3 & 75.6 & 85.5 & 75.7 & 68.9 & 57.5 \\
\hline & -0.2 & 13.2 & 9.0 & 13.2 & 8.8 & 12.2 & 5.9 & 18.6 & 11.0 & 18.2 & 11. & 15.4 & 9.2 & 25.8 & 16.1 & 25.9 & 16.4 & 21.1 & 11.8 \\
\hline & 0.2 & 11.5 & 6.0 & 12.0 & 6.1 & 11.7 & 6.6 & 16.4 & 9.7 & 16.0 & 9.3 & 15.9 & 8.5 & 23.3 & 14.0 & 23.6 & 13.9 & 20.1 & 12.4 \\
\hline & 0.5 & 24.7 & 15.4 & 25.2 & 15.0 & 22.1 & 13.6 & 51.9 & 38.6 & 51.9 & 38. & 41.3 & 30.6 & 78.5 & 67.6 & 78.6 & 67.9 & 66.0 & 54.3 \\
\hline & 1.0 & 67.9 & 55.8 & 67.1 & 55.3 & 58.6 & 45.8 & 97.5 & 95.1 & 97.5 & 95. & 92.7 & 88.8 & 100.0 & 99.9 & 100.0 & 99.9 & 99.9 & 99.6 \\
\hline \multirow[t]{6}{*}{$\overline{(1.0,1.0)}$} & $\begin{array}{c}-1.0 \\
\end{array}$ & 76.2 & 65.6 & 76.5 & 66.4 & 61.8 & 48.5 & 98.4 & 96.3 & 98.1 & 96. & 94.3 & 91.0 & 100.0 & 100.0 & 100.0 & 100.0 & 100.0 & 100.0 \\
\hline & -0.5 & 31.6 & 20.4 & 31.6 & 20.9 & 24.0 & 14.4 & 57.6 & 44.2 & 57.6 & 44.7 & 42.5 & 31.9 & 86.1 & 77.3 & 86.5 & 77.6 & 68.9 & 57.5 \\
\hline & -0.2 & 14.4 & 9.0 & 14.4 & 9.2 & 12.2 & 5.9 & 19.1 & 11.9 & 18.8 & 11. & 15.4 & 9.2 & 26.4 & 16.9 & 26.7 & 16.9 & 21.1 & 11.8 \\
\hline & 0.2 & 11.1 & 5.4 & 11.1 & 5.4 & 11.7 & 6.6 & 15.0 & 8.3 & 15.2 & 7.9 & 15.9 & 8.5 & 21.6 & 13.0 & 21.8 & 12.9 & 20.1 & 12.4 \\
\hline & 0.5 & 22.5 & 12.0 & 22.3 & 12.1 & 22.1 & 13.6 & 48.9 & 36.0 & 48.7 & 35. & 41.3 & 30.6 & 76.5 & 64.9 & 76.3 & 64.8 & 66.0 & 54.3 \\
\hline & 1.0 & 63.9 & 49.6 & 62.6 & 48.2 & 58.6 & 45.8 & 97.0 & 94.1 & 96.9 & 94. & 92.7 & 88.8 & 100.0 & 99.9 & 100.0 & 99.9 & 99.9 & 99.6 \\
\hline
\end{tabular}

Notes : (i) 1000 iterations;

(ii) $G L R$, the generalized likelihood ratio test, $q_{n}$ and $q_{n}^{0}$, loss function-based tests;

(iii) $q_{n}$ is standardized by $S S R_{1}$, the sum of squared residuals of the nonparametric regression estimates, and $q_{n}^{0}$ is standardized by $S S R_{0}$, the sum of squared residuals of the null linear model;

(iv) The uniform kernel is used for $G L R, q_{n}$ and $q_{n}^{0}$; the bandwidth $h=S_{X} n^{-1 / 5}$, where $S_{X}$ is the sample standard deviation of $\left\{X_{t}\right\}_{t=1}^{n}$;

(v) The $q_{n}$ tests are based on the linex loss function: $d(z)=\frac{\beta}{\alpha^{2}}[\exp (\alpha z)-1-\alpha z]$;

(vi) DGP P.2, $Y_{t}=1+X_{t} 1\left(X_{t}>0\right)+(1+\theta) X_{t} 1\left(X_{t} \leq 0\right)+\varepsilon_{t}$, where $\left\{\varepsilon_{i}\right\} \sim$ i.i.d.N $(0,1)$. 
TABLE S.5. Empirical Powers of Tests Using Bootstrap Critical Values

\begin{tabular}{|c|c|c|c|c|c|c|c|c|c|c|c|c|c|c|c|c|c|c|c|}
\hline & & \multicolumn{18}{|c|}{ DGP P.3: Smooth Transition Regression } \\
\hline & & \multicolumn{2}{|c|}{$q_{n}$} & \multicolumn{2}{|c|}{ 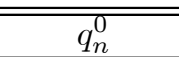 } & \multicolumn{2}{|c|}{ GLR } & \multicolumn{2}{|c|}{$q_{n}$} & \multicolumn{2}{|c|}{$q_{n}^{0}$} & \multicolumn{2}{|c|}{ GLR } & \multicolumn{2}{|c|}{$q_{n}$} & \multicolumn{2}{|c|}{$q_{n}^{0}$} & \multicolumn{2}{|c|}{$\overline{~ G L R}$} \\
\hline \multirow[t]{2}{*}{$(a, \beta)$} & $\theta$ & $10 \%$ & $5 \%$ & $10 \%$ & $5 \%$ & $10 \%$ & $5 \%$ & $10 \%$ & $5 \%$ & $10 \%$ & $5 \%$ & $10 \%$ & $5 \%$ & $10 \%$ & $5 \%$ & $10 \%$ & $5 \%$ & $10 \%$ & $5 \%$ \\
\hline & & \multicolumn{6}{|c|}{$n=100$} & \multicolumn{6}{|c|}{$n=250$} & \multicolumn{6}{|c|}{$n=500$} \\
\hline \multirow[t]{5}{*}{$(0.0,1.0)$} & -1.0 & 47.1 & 33.3 & 46.3 & 33.6 & 39.3 & 29.2 & 87.6 & 80.4 & 87.3 & 78.6 & 75.5 & 64.3 & 99.4 & 99.1 & 99.4 & 99.1 & 97.5 & 95.3 \\
\hline & -0.5 & 18.0 & 12.3 & 17.5 & 12.0 & 18.3 & 10.6 & 36.0 & 25.2 & 35.7 & 24.8 & 29.9 & 19.4 & 61.1 & 49.2 & 61.0 & 49.2 & 61.7 & 47.6 \\
\hline & 0.5 & 20.3 & 12.0 & 21.0 & 12.0 & 16.5 & 9.7 & 38.0 & 27.0 & 37.7 & 27.0 & 29.3 & 19.9 & 57.3 & 45.6 & 57.3 & 46.3 & 45.6 & 34.2 \\
\hline & 1.0 & 51.7 & 38.7 & 51.5 & 39.0 & 41.4 & 29.6 & 87.0 & 78.6 & 87.2 & 79.0 & 77.2 & 67.2 & 99.1 & 98.2 & 99.1 & 98.2 & 96.8 & 94.0 \\
\hline & 1.5 & 83.2 & 74.8 & 82.8 & 75.1 & 73.2 & 61.6 & 99.5 & 98.7 & 99.4 & 98.6 & 98.2 & 96.2 & 100.0 & 100.0 & 100.0 & 100.0 & 100.0 & 100.0 \\
\hline \multirow[t]{5}{*}{$(0.2,1.0)$} & -1.0 & 48.5 & 35.3 & 48.1 & 35.9 & 39.3 & 29.2 & 88.1 & 80.7 & 88.0 & 80.3 & 75.5 & 64.3 & 99.5 & 99.1 & 99.5 & 99.1 & 97.5 & 95.3 \\
\hline & -0.5 & 19.1 & 12.7 & 18.8 & 12.7 & 18.3 & 10.6 & 37.3 & 26.7 & 37.3 & 26.2 & 29.9 & 19.4 & 62.3 & 50.7 & 62.2 & 50.6 & 47.6 & 35.6 \\
\hline & 0.5 & 19.5 & 11.0 & 20.1 & 10.4 & 16.5 & 9.7 & 36.2 & 26.3 & 36.5 & 26.1 & 29.3 & 19.9 & 55.9 & 44.5 & 56.3 & 44.7 & 45.6 & 34.2 \\
\hline & 1.0 & 49.8 & 36.6 & 49.6 & 36.7 & 41.4 & 29.6 & 86.1 & 78.0 & 86.5 & 77.7 & 77.2 & 67.2 & 99.1 & 98.1 & 99.1 & 98.2 & 96.8 & 94.0 \\
\hline & 1.5 & 82.1 & 72.6 & 81.8 & 73.2 & 73.2 & 61.6 & 99.3 & 98.5 & 99.3 & 98.5 & 98.2 & 96.2 & 100.0 & 100.0 & 100.0 & 100.0 & 100.0 & 100.0 \\
\hline \multirow[t]{5}{*}{$(0.5,1.0)$} & -1.0 & 51.6 & 38.9 & 51.5 & 38.4 & 39.3 & 29.2 & 89.0 & 82.2 & 89.2 & 82.8 & 75.5 & 64.3 & 99.5 & 99.1 & 99.5 & 99.2 & 97.5 & 95.3 \\
\hline & -0.5 & 19.7 & 13.3 & 19.7 & 13.6 & 18.3 & 10.6 & 39.3 & 28.5 & 39.6 & 27.9 & 29.9 & 19.4 & 63.1 & 52.5 & 63.2 & 52.8 & 47.6 & 61.7 \\
\hline & 0.5 & 18.3 & 9.7 & 18.1 & 8.9 & 16.5 & 9.7 & 34.1 & 23.7 & 34.2 & 23.6 & 29.3 & 19.9 & 54.3 & 43.1 & 54.9 & 43.0 & 45.6 & 34.2 \\
\hline & 1.0 & 46.6 & 33.7 & 45.6 & 32.9 & 41.4 & 29.6 & 84.5 & 74.8 & 85.2 & 75.3 & 77.2 & 67.2 & 99.0 & 97.8 & 99.0 & 97.8 & 96.8 & 94.0 \\
\hline & 1.5 & 80.0 & 69.4 & 79.2 & 69.0 & 73.2 & 61.6 & 99.1 & 98.4 & 99.1 & 98.4 & 98.2 & 96.2 & 100.0 & 100.0 & 100.0 & 100.0 & 100.0 & 100.0 \\
\hline \multirow[t]{5}{*}{$(1.0,1.0)$} & -1.0 & 54.6 & 42.9 & 54.6 & 41.7 & 39.3 & 29.2 & 89.8 & 84.5 & 89.9 & 94.6 & 75.5 & 64.3 & 99.6 & 99.2 & 99.5 & 99.2 & 97.5 & 95.3 \\
\hline & -0.5 & 22.0 & 14.9 & 22.5 & 14.7 & 18.3 & 10.6 & 41.5 & 29.6 & 41.7 & 30.4 & 29.9 & 19.4 & 64.9 & 54.7 & 64.8 & 55.4 & 47.6 & 61.7 \\
\hline & 0.5 & 16.1 & 8.2 & 15.1 & 8.0 & 16.5 & 9.7 & 30.6 & 20.0 & 30.0 & 20.0 & 29.3 & 19.9 & 51.8 & 39.7 & 51.8 & 39.4 & 45.6 & 34.2 \\
\hline & 1.0 & 41.6 & 28.2 & 40.9 & 28.1 & 41.4 & 29.6 & 82.6 & 71.6 & 82.9 & 71.5 & 77.2 & 67.2 & 98.9 & 97.4 & 98.8 & 97.4 & 96.8 & 94.0 \\
\hline & 1.5 & 75.6 & 63.2 & 74.4 & 62.2 & 73.2 & 61.6 & 98.8 & 97.7 & 98.8 & 97.7 & 98.2 & 96.2 & 100.0 & 100.0 & 100.0 & 100.0 & 100.0 & 100.0 \\
\hline
\end{tabular}

Notes : (i) 1000 iterations;

(ii) $G L R$, the generalized likelihood ratio test, $q_{n}$ and $q_{n}^{0}$, loss function-based tests;

(iii) $q_{n}$ is standardized by $S S R_{1}$, the sum of squared residuals of the nonparametric regression estimates, and $q_{n}^{0}$ is standardized by $S S R_{0}$, the sum of squared residuals of the null linear model;

(iv) The uniform kernel is used for $G L R, q_{n}$ and $q_{n}^{0}$; the bandwidth $h=S_{X} n^{-1 / 5}$, where $S_{X}$ is the sample standard deviation of $\left\{X_{t}\right\}_{t=1}^{n}$;

(v) The $q_{n}$ tests are based on the linex loss function: $d(z)=\frac{\beta}{\alpha^{2}}[\exp (\alpha z)-1-\alpha z]$;

(vi) DGP P.3, $Y_{t}=1+X_{t}+\left[1-F\left(X_{t}\right) \theta\right] X_{t}+\varepsilon_{t}, F\left(X_{t}\right)=\frac{1}{1+\exp \left(-X_{t}\right)}$, where $\left\{\varepsilon_{i}\right\} \sim$ i.i.d.N $(0,1)$. 